\title{
Classification of $\mathrm{SL}_{2}$ deformed Floquet Conformal Field Theories
}

\author{
Bo $\operatorname{Han}^{1}$ and Xueda Wen ${ }^{2}$ \\ ${ }^{1}$ Theory of Condensed Matter Group, Cavendish Laboratory, University of Cambridge \\ J. J. Thomson Avenue, Cambridge CB3 OHE, United Kingdom \\ ${ }^{2}$ Department of Physics, Massachusetts Institute of Technology, Cambridge, MA 02139, USA
}

\begin{abstract}
Classification of the non-equilibrium quantum many-body dynamics is a challenging problem in condensed matter physics and statistical mechanics. In this work, we study the basic question that whether a $(1+1)$ dimensional conformal field theory $(\mathrm{CFT})$ is stable or not under a periodic driving with $N$ non-commuting Hamiltonians. Previous works showed that a Floquet (or periodically driven) CFT driven by certain $\mathrm{SL}_{2}$ deformed Hamiltonians exhibit both non-heating (stable) and heating (unstable) phases. In this work, we show that the phase diagram depends on the types of driving Hamiltonians. In general, the heating phase is generic, but the non-heating phase may be absent in the phase diagram. For the existence of the non-heating phases, we give sufficient and necessary conditions for $N=2$, and sufficient conditions for $N>2$. These conditions are composed of $N$ layers of data, with each layer determined by the types of driving Hamiltonians. Our results also apply to the single quantum quench problem with $N=1$.
\end{abstract}

\section{INTRODUCTION}

Non-equilibrium many-body dynamics have received extensive attention recently because they show exotic properties that are missing in static systems and also they can be realized in experiments such as optical lattice and cold atomic systems. For example, a periodic drive creates novel systems that may not have an equilibrium analog, such as Floquet topological phases ${ }^{1-14}$ and time crystals ${ }^{15-23}$. Moreover, a periodic drive is also one of the basic protocols to study non-equilibrium phenomena, such as localization-thermalization transitions, prethermalization, dynamical Casimir effect, etc. ${ }^{24-32}$

Although there are rich properties and applications in the time-dependent driving physics in quantum manybody systems, exactly solvable setups are very rare. In general, we need to resort to numerical methods that are limited to a small-system size. In this work, we are interested in a quantum $(1+1)$ dimensional conformal field theory (CFT), which may be viewed as the low energy effective field theory of a many-body system at the critical point. For $(1+1)$ D CFTs, the property of conformal invariance can be exploited to constrain the operator content of the critical theory ${ }^{33-35}$ which makes it tractable for the study of non-equilibrium dynamics, such as the quantum quench problems. ${ }^{36,37}$ For a timedependent driven CFT, however, relatively little is known on the analytical properties of the non-equilibrium dynamcis.

Recent study along this direction was initialized in Ref. 38 and 39, where two non-commuting Hamiltonians are used to drive the CFT periodically in time. One of the driving Hamiltonians is chosen as the uniform one, and the other is chosen by deforming the uniform one with a sine-square deformation (SSD), ${ }^{38,40-52}$ which we will introduce in detail shortly. In this periodically driven CFT (or Floquet CFT), it is found there are both heating and non-heating phases, separated by a critical (phase transition) line. One of the 'order parameters' characterizing the phase diagram is the time evolution of entanglement entropy. It was found that the entanglement entropy grows linearly in time in the heating phase, oscillates in the non-heating phase, and grows logarithmically in time at the phase transition. ${ }^{39}$

Later in Ref. 53, further interesting features were found in the same setup. For example, the total energy grows exponentially in time in the heating phase, oscillates in the non-heating phase, and grows polynomially in time at the phase transition. In particular, in the heating phase, it was found there are interesting emergent spatial structures during the driving: The (chiral and anti-chiral) energy-momentum densities form an array of 'peaks' in the real space (See also Ref. 54 for the study of the energy density distribution). In Ref. 53, it was also found that the entanglement pattern in a Floquet CFT is closely related to the energy-momentum density distributions. The main contribution of quantum entanglement in the Floquet CFT comes from those between nearby energymomentum density peaks of the same chirality.

Most recently, the types of driving sequences are generalized from periodic to quasi-periodic ${ }^{55,56}$ and random ones. ${ }^{57}$ On the one hand, it was found that the heating phase is generic in all these three types of driving sequences. In particular, the features as found in the periodic driving in Ref. 53 turn out to be also generic in the other two types of drivings. On the other hand, there are some new features in the phase diagrams of the quasi-periodic and random drivings. For example, in a quasi-periodically driven CFT with Fibonacci sequence, the non-heating phases form a Cantor set of measure zero, and the heating rates in the heating phases exhibit self-similarity structures. In the random driving, the driven CFT is generally in the heating phase, but with some isolated exceptional points (See Ref. 57 for more details). The mechanism of the heating phase in a randomly driven CFT is analogous to the Anderson localization in $(1+1)$ d disordered system. ${ }^{57}$ In short, the phase diagrams of time-dependent driven CFTs depend on the types of driving sequences.

In this work, by fixing the driving sequence to be a 
periodic one, we are interested in how the types of driving Hamiltonians affect the phase diagrams of a Floquet CFT. Let us specify the meaning of "Hamiltonian types" first. Considering a CFT defined on a circle of length $L$, the driving Hamiltonians we consider are of the following form:

$$
H_{\mathrm{CFT}}=H_{\text {chiral }}+H_{\text {anti-chiral }}
$$

i.e., one can decompose the total Hamiltonian as the sum of chiral and anti-chiral parts. In terms of the energymomentum tensor $T(x)$, we have

$$
H_{\text {chiral }}=\frac{1}{2 \pi} \int_{0}^{L} f(x) T(x) d x,
$$

where $f(x)$ is an envelope function which deforms the chiral energy-momentum density, and it is similar for the anti-chiral part, where the anti-chiral energy-momentum tensor is denoted as $\bar{T}(x)$. Here $T(x)$ and $\bar{T}(x)$ are related to the energy density $T_{00}$ and momentum density $T_{01}(x)$ as $T_{00}=\frac{1}{2 \pi}(T+\bar{T})$ and $T_{01}=\frac{1}{2 \pi}(T-\bar{T})$. For different driving Hamiltonians, we can choose different envelope functions $f(x)$. In this work, we are interested in the deformation of a single wavelength, with

$$
f(x)=\sigma^{0}+\sigma^{+} \cos \frac{2 \pi q x}{L}+\sigma^{-} \sin \frac{2 \pi q x}{L}, \quad q \in \mathbb{Z},
$$

where $\sigma^{0}$ and $\sigma^{ \pm}$are real numbers which characterize the deformation. With this deformation, the Hamiltonian can be written as

$$
H_{\text {chiral }}=\frac{2 \pi}{L}\left(\sigma^{0} L_{0}+\sigma^{+} L_{q,+}+\sigma^{-} L_{q,-}\right)-\frac{\pi c}{12 L},
$$

where we have defined $L_{q,+}:=\frac{1}{2}\left(L_{q}+L_{-q}\right)$ and $L_{q,-}:=$ $\frac{1}{2 i}\left(L_{q}-L_{-q}\right)$, with $L_{n}:=\frac{c}{24} \delta_{n, 0}+\frac{L}{2 \pi} \int_{0}^{L} \frac{d x}{2 \pi} e^{i \frac{2 \pi n}{L} x} T(x)$ being the generators of Virasoro algebra, ${ }^{35}$ and $c$ is the central charge. One can find that $H_{\text {chiral }}$ in (1.4) is composed of three generators that generate the $\mathrm{SL}^{(q)}(2, \mathbb{R})$ group, which is isomorphic to the $q$-fold cover of $\operatorname{SL}(2, \mathbb{R})^{58}$. For this reason, we call the periodically driven CFT with the driving Hamiltonians in (1.1)-(1.3) as the $\mathrm{SL}_{2}$ deformed Floquet CFT. In general, the Hamiltonians in (1.4) can be classified into three types based on the quadratic Casimir: ${ }^{43,44,51}$

$$
c^{(2)}:=-\left(\sigma^{0}\right)^{2}+\left(\sigma^{+}\right)^{2}+\left(\sigma^{-}\right)^{2} .
$$

Different types of Hamiltonians can be classified as follows:

$$
\begin{array}{|l|l|l|l|}
\hline \text { Quadratic Casimir } & c^{(2)}<0 & c^{(2)}=0 & c^{(2)}>0 \\
\hline \text { Hamiltonian Type } & \text { Elliptic } & \text { Parabolic } & \text { Hyperbolic } \\
\hline
\end{array}
$$

The reason we use these terminologies will be clear when we discuss operator evolutions in Sec. II. Therein, we will see that the operator evolutions governed by different Hamiltonian types correspond to the Möbius transformations of hyperbolic/parabolic/elliptic types. An intuitive picture to understand the difference of these three

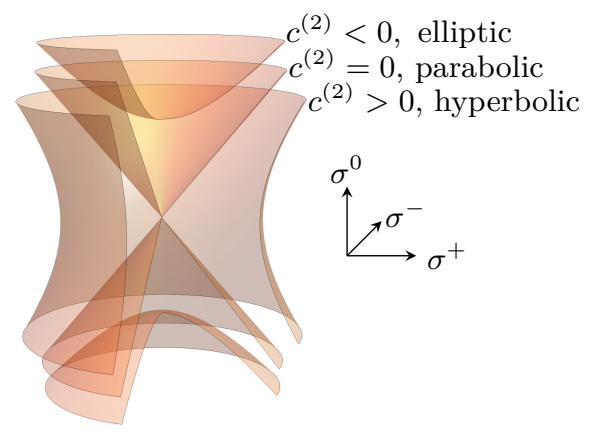

FIG. 1. Different types of manifolds determined by Eq.(1.5) with different quadratic Casimir $c^{(2)}$. Each single point on the manifold specifies a deformed Hamiltonian through (1.2) and (1.3). Any point on the manifold is $\mathrm{SL}(2, \mathbb{R})$ equivalent to arbitrary points on the same manifold.

Hamiltonian types is to consider the manifold determined by (1.5) with different signs of $c^{(2)}$, as shown in Fig. 1. Recently, the properties of energy spectrum for different types of Hamtiltonians have been studied in literature. ${ }^{43-46,51}$ As a remark, the specific choice of $q=1$, $\sigma^{0}=-\sigma^{+}=1 / 2$ and $\sigma^{-}=0$ in (1.3) corresponds to the CFT with SSD, as used in the simplest setup of a Floquet CFT in Ref. 39.

Our motivation in this work is to classify the nonequilibrium dynamics in a Floquet CFT with all possible choices of Hamiltonian types in (1.6). More specifically, during the periodic driving, we choose $N(N \geq 2)$ noncommuting Hamiltonians, with each Hamiltonian specified by a certain $\left(\sigma^{0}, \sigma^{+}, \sigma^{-}\right)$in (1.4). What we mean by 'classification' is to determine whether there are heating phases/non-heating phases/phase transitions in the phase diagram. The main results we found can be summarized as follows. For arbitrary choices of Hamiltonian types, there are always heating phases in the Floquet CFT, i.e., the heating phase is generic. The non-heating phases, however, do not always exist in the phase diagram. When there are $N=2$ driving Hamiltonians, we give the sufficient and necessary conditions for the existence of non-heating phases in the phase diagram, as shown in Table. I. For $N>2$, we give sufficient conditions for the existence of non-heating phases, as presented in Sec. III D. These conditions can be summarized as follows: There are in total $N$ layers of data. At each layer $n$, we pick arbitrary $n$ driving Hamiltonians by keeping the time order of driving. If there is at least one elliptic Hamiltonian in $\left\{H_{i_{1}}, H_{i_{2}}, \cdots, H_{i_{n}}\right\}$, then there must exist non-heating phases. If all the Hamiltonians in $\left\{H_{i_{1}}, H_{i_{2}}, \cdots, H_{i_{n}}\right\}$ are non-elliptic (either parabolic or hyperbolic), then the following condition ensures the existence of non-heating phases:

$$
\exists \eta_{n}<0, \quad n=1,2, \cdots, N
$$

where $\eta_{n}$ is the indicator as defined in (3.25). It is noted that $\eta_{n}$ is only determined by the vectors $\left(\sigma_{i}^{0}, \sigma_{i}^{+}, \sigma_{i}^{-}\right)$ 
that characterize these driving Hamiltonians. One can find there are in total $2^{N}-1$ conditions for $N$ driving Hamiltonians. If at least one of these conditions is satisfied, then there must exist non-heating phases in the phase diagram. We suspect that these conditions are also necessary, i.e., if there exists a non-heating phase in the phase diagram, then at least one of the $2^{N}-1$ conditions mentioned above should be satisfied, although we have not yet found a mathematical proof when $N>2$.

The structure of this paper is organized as follows. In Sec. II, we introduce the setup for a general Floquet CFT with $\mathrm{SL}_{2}$ deformations. Then we study the operator evolution corresponding to different Hamiltonian types, based on which the entanglement/energymomentum evolution can be obtained. In Sec. III, we study how the phase diagrams depend on the types of $N$ driving Hamiltonians in a Floquet CFT. We give sufficient conditions for the existence of non-heating phases for arbitrary $N$, and illustrate these conditions with examples $N=1,2$, and 3 . We give some discussions and conclude in Sec. IV. There are also several appendices focusing on the detailed features of the phase diagrams with different types of driving Hamiltonians.

\section{II. $\mathrm{SL}_{2}$ DEFORMED FLOQUET CFT}

\section{A. Setup}

The setup we consider is based on a $(1+1)$ dimensional CFT on a circle of length $L$ with periodic boundary conditions. The driving Hamiltonians we choose are of the form in Eqs. (1.1)-(1.3). Then we study a timedependent driving with a discrete and periodic sequence:

$$
\left|\Psi_{n}\right\rangle=\left(U_{N} \cdots U_{2} \cdot U_{1}\right)^{m}\left|\Psi_{0}\right\rangle, \quad \text { with } \quad U_{j}=e^{-i H_{j} T_{j}},
$$

where $n=N m$, and $T_{j}$ is the time duration of driving with Hamiltonian $H_{j}$. Within each period, there are in general $N$ (possibly) different driving Hamiltonians. Here the initial state $\left|\Psi_{0}\right\rangle$ may be taken as the ground state of a uniform CFT Hamiltonian

$$
H_{0}=\frac{1}{2 \pi} \int_{0}^{L} T(x) d x+\text { anti-chiral part. }
$$

It is noted that the initial state can also be chosen as an excited state or even a thermal ensemble at finite temperature. ${ }^{59}$ Each driving Hamiltonian $H_{i}$ in $(2.1)$ can be chosen with different envelope functions $f_{i}(x)$ in (1.3), which are characterized by a triple $\left(\sigma_{i}^{0}, \sigma_{i}^{+}, \sigma_{i}^{-}\right)$. Our goal is to classify the non-equilibrium dynamics and phase diagrams with respect to the types of $H_{i}(i=1, \cdots, N)$ in the $N$ dimensional parameter space spanned by

$$
\left\{\left(\frac{T_{1}}{l}, \cdots, \frac{T_{N}}{l}\right) \mid 0<\frac{T_{i}}{l}<\infty, i=1, \cdots, N\right\},
$$

where $l:=L / q$ is the wavelength of deformation in $f(x)$ [see Eq.(1.3)].
As a remark, in the above discussions, we only specify the deformation of the chiral part of the Hamiltonian, which are characterized by the vector $\left(\sigma_{i}^{0}, \sigma_{i}^{+}, \sigma_{i}^{-}\right)$. The deformation of the anti-chiral parts are characterized by another independent vector $\left(\sigma_{i}^{0 \prime}, \sigma_{i}^{+\prime}, \sigma_{i}^{-\prime}\right)$, because we choose periodic boundary conditions and the chiral and anti-chiral modes are decoupled from each other. In the following study, without loss of generality, we will focus on the deformation of the chiral parts. The analysis of the anti-chiral parts can be performed in the same way.

\section{B. Hamiltonian types and operator evolution}

To understand the non-equilibrium dynamics under the driving in Eq. (2.1), we first study the operator evolution, based on which the time evolution of correlation functions such as the entanglement entropy and the energy-momentum density can be obtained ${ }^{38,39,53,54}$.

Let us start from driving the CFT with a single Hamiltonian $H$ with a time duration $t$. We consider the method as used in Ref. 39, which we briefly sketch as follows. In the Euclidean spacetime, the correlation function $\langle\Psi(t)|\mathcal{O}(x)| \Psi(t)\rangle=\left\langle G\left|e^{H \tau} \mathcal{O}(x) e^{-H \tau}\right| G\right\rangle=$ $\langle G|\mathcal{O}(x, \tau)| G\rangle$, where $\tau=i t$, can be considered as the path integral on a $w$-cylinder with the operator $\mathcal{O}$ inserted at $(x, \tau)$, as depicted in Fig. 2. This cylinder can be mapped to a $q$-sheet Riemann surface with a conformal map $z=e^{i \frac{2 \pi q w}{L}}$. Then the Hamiltonian in Eqs.(1.1)(1.3) can be written as $H=H^{(z)}+\bar{H}^{(\bar{z})}$, where

$$
\begin{aligned}
H^{(z)}=\frac{2 \pi}{l} \oint & \frac{d z}{2 \pi i}\left[\sigma^{0} z+\frac{1}{2}\left(\sigma^{+}-i \sigma^{-}\right) z^{2}\right. \\
& \left.+\frac{1}{2}\left(\sigma^{+}+i \sigma^{-}\right)\right] T(z)-\frac{\sigma^{0} \pi c}{12 l} .
\end{aligned}
$$

One can further perform a Möbius transformation $z=$ $(\mathfrak{a} \widetilde{z}+\mathfrak{b}) /(\mathfrak{c} \widetilde{z}+\mathfrak{d})$, where $\mathfrak{a}=-\frac{i}{2}, \mathfrak{b}=\frac{\sigma^{+}+i \sigma^{-}}{\sqrt{c^{(2)}}}, \mathfrak{c}=$ $\frac{-\sqrt{c^{(2)}}+i \sigma^{0}}{2\left(\sigma^{+}+i \sigma^{-}\right)}$, and $\mathfrak{d}=-\frac{\sigma^{0}+i \sqrt{c^{(2)}}}{\sqrt{c^{(2)}}}$. Then the Hamiltonian defined on the $q$-sheet $\widetilde{z}$ Riemann surface is of the simple form:

$$
H^{(\widetilde{z})}=-\frac{2 \pi i \sqrt{c^{(2)}}}{l} \oint \frac{d \widetilde{z}}{2 \pi i} \widetilde{z} T(\widetilde{z})-\frac{\sigma^{0} \pi c}{12 l} .
$$

Several remarks here. First, from (2.5), one can already see the difference for positive and negative $c^{(2)}$. The choice of branch cut of $\sqrt{\cdots}$ will not affect the results of operator evolution as presented later in Eqs.(2.8), (2.9), and (2.10). Second, for $c^{(2)}=0$, the expression in (2.5) is not well defined. To obtain the operator evolution, one can do the calculation by keeping nonzero $c^{(2)}$ and take the limit $c^{(2)} \rightarrow 0$ for either $c^{(2)}>0$ or $c^{(2)}<0$ in the last step. ${ }^{39,45,46,60}$

On the $q$-sheet $\widetilde{z}$ Riemann surface, the operator evolution becomes a dilatation: $e^{H^{(\widetilde{z})}} \mathcal{O}(\widetilde{z}, \bar{z}) e^{-H^{(\widetilde{z})} \tau}=$ 


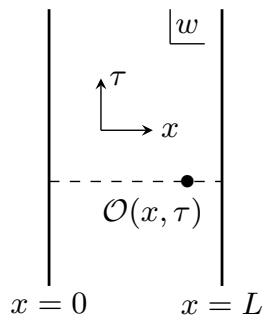

FIG. 2. Path integral representation of the correlation function $\langle G|\mathcal{O}(x, \tau)| G\rangle$ in a CFT with periodical boundary conditions. Here $x=0$ and $x=L$ are identified.

$\lambda^{h} \mathcal{O}(\lambda \widetilde{z}, \bar{z})$, where $\lambda=e^{-\frac{2 \pi i \sqrt{c^{(2)}}}{l}} \tau$, and $h$ is the conformal dimension of the operator $\mathcal{O}$. Then by mapping back to the $z$-surface, one can find the operator evolves as

$$
e^{H^{(z)} \tau} \mathcal{O}(z, \bar{z}) e^{-H^{(z)} \tau}=\left(\frac{\partial z^{\prime}}{\partial z}\right)^{h} \mathcal{O}\left(z^{\prime}, \bar{z}^{\prime}\right) .
$$

By doing an analytical continuation $\tau=i t$, where $t$ the time duration of driving, one has

$$
z^{\prime}=\frac{\alpha z+\beta}{\beta^{*} z+\alpha^{*}}=: M \cdot z, \quad M=\left(\begin{array}{cc}
\alpha & \beta \\
\beta^{*} & \alpha^{*}
\end{array}\right) \in \mathrm{SU}(1,1) .
$$

Note that $\mathrm{SU}(1,1) \cong \mathrm{SL}(2, \mathbb{R})$, which is as expected since the three generators $L_{0}$ and $L_{q, \pm}$ in Eq. (1.4) generate the $\mathrm{SL}^{(q)}(2, \mathbb{R})$ group. Depending on the types of driving Hamiltonians in $(1.6)$, the $\mathrm{SU}(1,1)$ matrices in Eq.(2.7) have different expressions as follows:

1. Elliptic $\left(c^{(2)}<0\right)$ :

$$
\left\{\begin{array}{l}
\alpha=\cos \left(\frac{\pi \mathcal{C} t}{l}\right)+i \frac{\sigma^{0}}{\mathcal{C}} \sin \left(\frac{\pi \mathcal{C} t}{l}\right), \\
\beta=i \frac{\sigma^{+}+i \sigma^{-}}{\mathcal{C}} \sin \left(\frac{\pi \mathcal{C} t}{l}\right) .
\end{array}\right.
$$

2. Parabolic $\left(c^{(2)}=0\right)$ :

$$
\left\{\begin{array}{l}
\alpha=1+i \frac{\sigma^{0} \pi t}{l}, \\
\beta=i \frac{\left(\sigma^{+}+i \sigma^{-}\right) \pi t}{l} .
\end{array}\right.
$$

3. Hyperbolic $\left(c^{(2)}>0\right)$ :

$$
\left\{\begin{array}{l}
\alpha=\cosh \left(\frac{\pi \mathcal{C} t}{l}\right)+i \frac{\sigma^{0}}{\mathcal{C}} \sinh \left(\frac{\pi \mathcal{C} t}{l}\right), \\
\beta=i \frac{\sigma^{+}+i \sigma^{-}}{\mathcal{C}} \sinh \left(\frac{\pi \mathcal{C} t}{l}\right) .
\end{array}\right.
$$

In all these three cases, we have defined the real number:

$$
\mathcal{C}:=\sqrt{\left|-\left(\sigma^{0}\right)^{2}+\left(\sigma^{+}\right)^{2}+\left(\sigma^{-}\right)^{2}\right|} .
$$

One can find that for different types of Hamiltonians, the corresponding Möbius transformations are qualitatively different. It is well known that there are in total three types of $\mathrm{SU}(1,1)$ matrices (See, e.g., Ref. 61) depending on the value of their traces: For $|\operatorname{Tr}(M)|<2,=2$, and $>2$, the corresponding $\mathrm{SU}(1,1)$ matrices are called elliptic, parabolic, and hyperbolic matrices, respectively. It is straightforward to check that for a general time duration $t$, where $t>0$, the Möbius transformations in Eqs. (2.8), (2.9), and (2.10) are elliptic, parabolic, and hyperbolic, respectively. For this reason, we denote the corresponding Hamiltonian types in (1.6).

Now let us consider an $\mathrm{SL}_{2}$ deformed Floquet CFT with $N$ driving Hamiltonians. Then the operator evolution after each period is determined by $z_{N}=\Pi_{N} \cdot z$ (and similarly for the anti-holomorphic part), where

$$
\Pi_{N}=M_{1} \cdots M_{N}=:\left(\begin{array}{cc}
\alpha_{N} & \beta_{N} \\
\beta_{N}^{*} & \alpha_{N}^{*}
\end{array}\right) \in \mathrm{SU}(1,1) .
$$

The operator evolution after $m$ periods of driving is determined by $\Pi_{N}$ as

$$
z_{n}=\left(\Pi_{N}\right)^{m} \cdot z, \quad n=m N .
$$

Then the phase diagram of the Floquet CFT is determined by $\left|\operatorname{Tr}\left(\Pi_{N}\right)\right|$ as follows: ${ }^{39,55}$

$$
\begin{cases}\left|\operatorname{Tr}\left(\Pi_{N}\right)\right|<2, & \text { non-heating phase, } \\ \left|\operatorname{Tr}\left(\Pi_{N}\right)\right|=2, & \text { phase transition, } \\ \left|\operatorname{Tr}\left(\Pi_{N}\right)\right|>2, & \text { heating phase. }\end{cases}
$$

As studied in Ref. 39, the value of $\left|\operatorname{Tr}\left(\Pi_{N}\right)\right|$ in (2.14) determines the trajectories of operator evolutions. For $\left|\operatorname{Tr}\left(\Pi_{N}\right)\right|<2$, the operator will rotate along the circle all the way; for $\left|\operatorname{Tr}\left(\Pi_{N}\right)\right|=2$, the operator will approach a fixed point polynomially fast in time; for $\left|\operatorname{Tr}\left(\Pi_{N}\right)\right|>2$, the operator will approach a fixed point exponentially fast in time. As we will see in the next subsection, the entanglement/energy-momentum density evolution will exhibit qualitatively different features in these different regimes.

\section{Entanglement and energy-momentum evolution}

Once we know the operator evolution in (2.7), we can study the time evolution of correlation functions. The 'order parameters' we use to distinguish different dynamical phases are the entanglement entropy and energymomentum evolutions, which can be viewed as the correlation functions of twist operators and energy-momentum tensor, respectively. Some of related details can also be found in Refs. 38, 39, 53, and 55 .

Let us consider the time evolution of entanglement entropy first. With the twist-operator approach, ${ }^{62,63}$ 
one can find the $\alpha$-th Renyi entropy of the subsystem $A=\left(x_{1}, x_{2}\right)$ as

$$
S_{A}^{(\alpha)}=\frac{1}{1-\alpha} \log \left\langle\Psi_{n}\left|\mathcal{T}\left(w_{1}, \bar{w}_{1}\right) \overline{\mathcal{T}}\left(w_{2}, \bar{w}_{2}\right)\right| \Psi_{n}\right\rangle
$$

where $\left|\Psi_{n}\right\rangle$ is the wavefunction in (2.1) by choosing the initial state $\left|\Psi_{0}\right\rangle$ as the ground state of the uniform Hamiltonian $H_{0}$ in $(2.2)$, and $\mathcal{T}(\overline{\mathcal{T}})$ are twist operators with conformal dimensions $h=\bar{h}=\frac{c}{24}(\alpha-1 / \alpha)$. As studied in the previous subsection, to evaluate the correlation function of the twist operators, we first map the $w$ cylinder to the $q$-sheet Riemann surface $z$ by $z=e^{i \frac{2 \pi q w}{L}}$, where the evolution of $z_{1}\left(\bar{z}_{1}\right) z_{2}\left(\bar{z}_{2}\right)$ are governed by Möbius transformations in Eq. (2.13). Next, we map the $z$-Riemann surface to a complex plane $\zeta$ by $\zeta=z^{1 / q}$. One can find that

$$
\begin{aligned}
& \left\langle\Psi_{n}\left|\mathcal{T}\left(w_{1}, \bar{w}_{1}\right) \overline{\mathcal{T}}\left(w_{2}, \bar{w}_{2}\right)\right| \Psi_{n}\right\rangle \\
= & \prod_{i=1,2}\left(\frac{\partial \zeta_{i}}{\partial w_{i}}\right)^{h} \prod_{i=1,2}\left(\frac{\partial \bar{\zeta}_{i}}{\partial \bar{w}_{i}}\right)^{\bar{h}}\left\langle\mathcal{T}\left(\zeta_{1}, \bar{\zeta}_{1}\right) \overline{\mathcal{T}}\left(\zeta_{2}, \bar{\zeta}_{2}\right)\right\rangle_{\zeta}
\end{aligned}
$$

where we have $w_{i}=x_{i}+i \tau=x_{i}$. For a general choice of the subsystem $A$, the expression of $S_{A}^{(\alpha)}$ is complicated. ${ }^{39}$ Here, for simplicity we choose the subsystem $A$ as a unit cell with $\left(x_{1}, x_{2}\right)=(k l,(k+1) l)$ where $k \in \mathbb{Z}$. Then it is straightforward to find that

$$
S_{A}(n)-S_{A}(0)=\frac{c}{3}\left(\log \left|\alpha_{n}+\beta_{n}\right|+\log \left|\alpha_{n}^{\prime}+\beta_{n}^{\prime}\right|\right),
$$

where $\alpha_{n}^{\prime}\left(\beta_{n}^{\prime}\right)$ corresponds to the driving effect in the anti-chiral parts and we have considered $S_{A}=$ $\lim _{\alpha \rightarrow 1} S_{A}^{(\alpha)}$. Here $\alpha_{n}$ and $\beta_{n}$ are the matrix elements in $\left(\Pi_{N}\right)^{m}$ in $(2.13)$, i.e.,

$$
\left(\begin{array}{cc}
\alpha_{n} & \beta_{n} \\
\beta_{n}^{*} & \alpha_{n}^{*}
\end{array}\right)=\left(\begin{array}{cc}
\alpha_{N} & \beta_{N} \\
\beta_{N}^{*} & \alpha_{N}^{*}
\end{array}\right)^{m}, \quad n=m N
$$

As a remark, if one studies the entanglement entropy of $A$ by shifting a half unit cell, i.e., $A=\left[\left(k+\frac{1}{2}\right) l,\left(k+\frac{3}{2}\right) l\right]$ where $k \in \mathbb{Z}$, then one can find that

$$
S_{A}(n)-S_{A}(0)=\frac{c}{3}\left(\log \left|\alpha_{n}-\beta_{n}\right|+\log \left|\alpha_{n}^{\prime}-\beta_{n}^{\prime}\right|\right) .
$$

The difference between Eq. (2.17) and Eq. (2.19) reflects the fact that the system is driven in a non-uniform way.

Next, let us consider the energy-momentum density evolution. With the operator evolution in (2.13), one has

$$
U^{\dagger} T(z) U=\left(\frac{\partial z^{\prime}}{\partial z}\right)^{2} T\left(z^{\prime}\right)+\frac{c}{12} \operatorname{Sch}\left\{z^{\prime}, z\right\}
$$

where $U=\left(U_{N} \cdots U_{2} \cdot U_{1}\right)^{m}$ [see Eq. (2.1)], and the second term represents the Schwarzian derivative. One can obtain the expectation value of the chiral energymomentum tensor density as follows ${ }^{53}$

$$
\frac{1}{2 \pi}\langle T(x, n)\rangle=-\frac{q^{2} \pi c}{12 L^{2}}+\frac{\pi c}{12 L^{2}} \cdot\left(q^{2}-1\right) \cdot \frac{1}{\left|\alpha_{n} \cdot z+\beta_{n}\right|^{4}}
$$

where $z=e^{\frac{2 \pi i q x}{L}}$, and $\alpha_{n}$ and $\beta_{n}$ are those defined in Eq.(2.18). By integrating over the energy-momentum density, one can obtain the total energy as

$$
\begin{aligned}
E(n) & =\frac{1}{2 \pi} \int_{0}^{L}\langle T(x, n)+\bar{T}(x, n)\rangle d x \\
& =-\frac{q^{2} \pi c}{6 L}+\frac{\pi c}{12 L}\left(q^{2}-1\right) \cdot\left(\left|\alpha_{n}\right|^{2}+\left|\beta_{n}\right|^{2}+\left|\alpha_{n}^{\prime}\right|^{2}+\left|\beta_{n}^{\prime}\right|^{2}\right)
\end{aligned}
$$

Now let us comment on how different types of Möbius transformations in Eqs. (2.8), (2.9), and (2.10) result in different behaviors of entanglement/energy evolution. Based on the analysis in Refs. 39 and 55, the norm $\left|\alpha_{n}\right|$ $\left(\left|\beta_{n}\right|\right)$ will grow exponentially/polynomically/oscillate in time when the corresponding Möbius transformations within one period is hyperbolic/parabolic/elliptic. Based on the expressions of entanglement and energymomentum density evolution in Eqs. (2.17) and (2.22), one can find that in general the entanglement entropy will grow linearly/grow logarithmically/oscillate in time, and the total energy will grow exponentially/grow polynomially/oscillate in time accordingly. We will see an illustrating example later in Sec. III A.

\section{CLASSIFYING THE $\mathrm{SL}_{2}$ DEFORMED FLOQUET CFT}

Now we come to the main section of this work: we study the conditions for the existence of heating and nonheating phases in the phase diagrams when there are $N$ driving Hamiltonians. It is found that there are always heating phases in the phase diagram. For the non-heating phases, we will give conditions for their existence in the phase diagram. Our conditions are both sufficient and necessary for $N=1$ and $N=2$, and are sufficient for $N>2$. We will illustrate these conditions by considering $N=1,2$, and 3 , and then give the general results for arbitrary $N$.

\section{A. $N=1$}

As a warm up, let us first consider the simplest case with $N=1$, i.e., there is only one driving Hamiltonian $H_{1}$. This case corresponds to a single quantum quench rather than a Floquet CFT. Here we consider this simple case to illustrate how different Hamiltonian types in (1.6) determine different behaviors of entanglement evolution.

It is noted that in Ref. 38, the single quantum quench was studied for some specific Hamiltonians $H_{1}$ with $c^{(2)}=0$ and $c^{(2)}<0$, respectively. It was found that 
the entanglement entropy grows logarithmically in time for $c^{(2)}=0$, and simply oscillates in time for $c^{(2)}<0$. Here we consider the more general Hamiltonians as described in Eqs. (1.2) and (1.3).

As discussed in the previous subsections, different types of Hamiltonians will determine different kinds of operator evolution in Eqs. (2.8), (2.9) and (2.10), which further determine the entanglement and energymomentum density through Eqs. (2.17) and (2.21).

Let us consider the entanglement entropy evolution for example. Considering the subsystem $A=[k l,(k+1) l]$, where $k \in \mathbb{Z}$, it can be found that in the long time driving limit (i.e., $\mathcal{C} t / l \gg 1$ in the hyperbolic case, and $t / l \gg$ 1 in the parabolic case), the entanglement entropy will grow linearly in time for $c^{(2)}>0$ (hyperbolic case), grow logarithmically in time for $c^{(2)}=0$ (parabolic case), and oscillate in time for $c^{(2)}<0$ (elliptic case) in the following way:

$$
S_{A}(t)-S_{A}(0) \simeq \begin{cases}\frac{\pi c}{6} \cdot \frac{\mathcal{C} t}{l}, & c^{(2)}>0, \\ \frac{c}{3} \log \frac{\pi t}{l}, & c^{(2)}=0, \\ \frac{c}{6} \log \left|a+b \sin \left(\frac{2 \pi \mathcal{C} t}{l}+\phi\right)\right|, & c^{(2)}<0,\end{cases}
$$

where $\mathcal{C}$ is defined in Eq. (2.11). The real numbers $a$, $b$, and $\phi$ depend on the parameters $\left(\sigma^{0}, \sigma^{+}, \sigma^{-}\right)$. One can refer to Appendix. A for a complete expression of the entanglement entropy evolution with arbitrary time duration $t>0$.

In short, the non-equilibrium dynamics for $N=1$ is only determined by the Hamiltonian types in (1.6).

\section{B. $N=2$}

Now we consider the properties of the phase diagram in the case of $N=2$, i.e., there are two noncommuting Hamiltonians. Since there are three possibilities of Hamiltonian types for $H_{1}\left(H_{2}\right)$, we have in total six different unordered pairings of $H_{1}$ and $H_{2}$ (See Table I).

For later use, let us define the Casimir vector that characterizes the $\mathrm{SL}_{2}$ deformed Hamiltonian in (1.3):

$$
\mathcal{C}_{i}=\left(\sigma_{i}^{0}, \sigma_{i}^{+}, \sigma_{i}^{-}\right)
$$

as well as the product of two Casimir vectors

$$
\mathcal{C}_{i} \cdot \mathcal{C}_{j}:=-\sigma_{i}^{0} \sigma_{j}^{0}+\sigma_{i}^{+} \sigma_{j}^{+}+\sigma_{i}^{-} \sigma_{j}^{-} .
$$

Our strategy to determine the phase diagram in the parameter space spanned by $\left\{\left(T_{1} / l, T_{2} / l\right) \mid 0<T_{1} / l, T_{2} / l<\right.$ $\infty\}$ can be briefly summarized as follows. The effect of driving with Hamtiltonian $H_{1}\left(H_{2}\right)$ for a time duration $T_{1}\left(T_{2}\right)$ are represented by a $\mathrm{SU}(1,1)$ matrix $M_{1}\left(M_{2}\right)$. Depending on the types of $H_{1}\left(H_{2}\right)$, the $\mathrm{SU}(1,1)$ matrix $M_{1}\left(M_{2}\right)$ takes the form in one of Eqs. (2.8), (2.9),
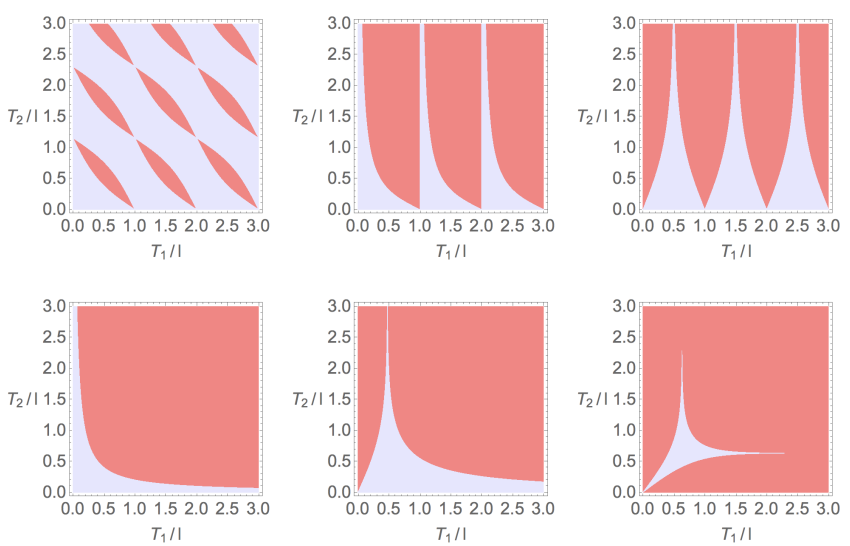

FIG. 3. Phase diagrams of a Floquet CFT with the both non-heating (in blue) and heating (in red) phases for the six kinds of pairings with $N=2$ in Table.I. The parameters are (from left to right, and then top to bottom): elliptic-elliptic with $\mathcal{C}_{1}=(1,0,0)$ and $\mathcal{C}_{2}=(1,0.5,0)$; elliptic-parabolic with $\mathcal{C}_{1}=(1,0,0)$ and $\mathcal{C}_{2}=(1,1,0)$; elliptic-hyperbolic with $\mathcal{C}_{1}=$ $(1,0,0)$ and $\mathcal{C}_{2}=(0,0.4,0)$; parabolic-parabolic with $\mathcal{C}_{1}=$ $(1,1,0)$ and $\mathcal{C}_{2}=(1,0,1)$; parabolic-hyperbolic with $\mathcal{C}_{1}=$ $(1,1,0)$ and $\mathcal{C}_{2}=(1,0.6,1)$; hyperbolic-hyperbolic with $\mathcal{C}_{1}=$ $(1,1.4,0)$ and $\mathcal{C}_{2}=(1,0,1.4)$.

and (2.10). Then the phase diagram is determined by $\left|\operatorname{Tr}\left(M_{1} \cdot M_{2}\right)\right|=\left|\operatorname{Tr}\left(M_{2} \cdot M_{1}\right)\right|$ based on Eq. (2.14).

Fig. 3 is a sample plot of the phase diagrams for the six different pairings of $H_{1}$ and $H_{2}$ in Table I. The parameters in $\mathcal{C}_{1}$ and $\mathcal{C}_{2}$ are chosen such that there are both heating and non-heating phases in the phase diagram. For arbitrary choices of $\mathcal{C}_{1}$ and $\mathcal{C}_{2}$, the heating phases are generic, but the non-heating phases may be absent. In Table I, we give the sufficient and necessary conditions for the existence of non-heating phases in the phase diagram. The details of derivations of these conditions can be found in Appendix B.

\begin{tabular}{|c|c|c|c|}
\hline$H_{1} H_{2}$ & Elliptic & Parabolic & Hyperbolic \\
\hline Elliptic & $\sqrt{ }$ & $\sqrt{ }$ & $\sqrt{ }$ \\
\hline Parabolic & $\sqrt{ }$ & $\mathcal{C}_{1} \cdot \mathcal{C}_{2}<0$ & $\mathcal{C}_{1} \cdot \mathcal{C}_{2}<0$ \\
\hline Hyperbolic & $\sqrt{ }$ & $\mathcal{C}_{1} \cdot \mathcal{C}_{2}<0$ & $1+\frac{\mathcal{C}_{1} \cdot \mathcal{C}_{2}}{\mathcal{C}_{1} \mathcal{C}_{2}}<0$ \\
\hline
\end{tabular}

TABLE I. Sufficient and necessary conditions for the existence of non-heating phases with $N=2$. " $\sqrt{ }$ " means the nonheating phases exist for arbitrary choices of non-commuting Hamiltonians $H_{1}$ and $H_{2}$ of the corresponding types.

Let us give several remarks on how to obtain the conditions in Table I. If at least one of the driving Hamiltonians is elliptic, then there must exist non-heating phases in the phase diagram. This can be straightforwardly understood as follows. Denoting the elliptic Hamiltonian as $H_{1}$, then the limit $T_{1} / l \neq 0$ and $T_{2} / l=0(j \neq i)$ corresponds to a single quench problem with $N=1$. In this case we have $\left|\operatorname{Tr}\left(M_{1} \cdot M_{2}\right)\right|<2$. Now we turn on $T_{2} / l$. As long as $T_{2} / l$ is small enough, we still have 


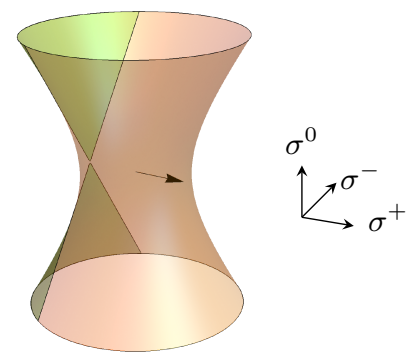

FIG. 4. $\frac{\mathcal{C}_{1}}{\mathcal{C}_{1}}=\left(\sigma_{1}^{0}, \sigma_{1}^{+}, \sigma_{1}^{-}\right)=(0,1,0)$ is fixed (the vector in black). The normalized vectors $\frac{\mathcal{C}_{2}}{\mathcal{C}_{2}}$ that satisfy the condition in Eq.(3.11) are in the region in green.

$\left|\operatorname{Tr}\left(M_{1} \cdot M_{2}\right)\right|<2$, i.e., the Floquet CFT is in a nonheating phase. Essentially, this condition is a quasi$(N-1)$ condition, since only $(N-1)$ driving Hamiltonians dominate while the left Hamiltonian plays little role. The five conditions labeled by ' $\sqrt{ }$ ' in Table I are all quasi- $(N-1)$ conditions, with $N=2$. The left four conditions are intrinsic- $N$ conditions, which can be expressed by one condition:

$$
\eta_{2}<0
$$

Here the indicator $\eta_{N=2}$ is constructed as follows. For each driving Hamiltonian $H_{j}$, we arrange a matrix $P_{j}$ in the following way. If $H_{j}$ is parabolic, then

$$
P_{j}=\left(\begin{array}{cc}
i \sigma_{j}^{0} & i\left(\sigma_{j}^{+}+i \sigma_{j}^{-}\right) \\
-i\left(\sigma_{j}^{+}-i \sigma_{j}^{-}\right) & -i \sigma_{j}^{0}
\end{array}\right) .
$$

If $H_{j}$ is hyperbolic, then the corresponding matrix is

$$
P_{j}=\left(\begin{array}{cc}
1+i \frac{\sigma_{j}^{0}}{\mathcal{C}_{j}} & i \frac{\left(\sigma_{j}^{+}+i \sigma_{j}^{-}\right)}{\mathcal{C}_{j}} \\
-i \frac{\left(\sigma_{j}^{+}-i \sigma_{j}^{-}\right)}{\mathcal{C}_{j}} & 1-i \frac{\sigma_{j}^{0}}{\mathcal{C}_{j}}
\end{array}\right) .
$$

Here $P_{j}$ are obtained based on the $\mathrm{SU}(1,1)$ matrix in (2.7) as follows. For parabolic $H_{j}$, one has

$$
P_{j}=\lim _{t / l \rightarrow \infty}\left[\left(\frac{\pi t}{l}\right)^{-1} M\right],
$$

with $M$ in (2.9). For hyperbolic $H_{j}$, one has

$$
P_{j}=\lim _{\mathcal{C} t / l \rightarrow \infty}\left[\left(\cosh \frac{\pi \mathcal{C} t}{l}\right)^{-1} M\right],
$$

with $M$ in (2.10). Then the indicator is defined as

$$
\eta_{2}:=\operatorname{Tr}\left(P_{1} \cdot P_{2}\right) .
$$

One can check explicitly that if at least one of the nonelliptic Hamiltonians is parabolic, then the condition in (3.4) becomes

$$
\mathcal{C}_{1} \cdot \mathcal{C}_{2}<0
$$

where the product of two Casimir vectors is defined in (3.3). If both of the two driving Hamiltonians are hyperbolic, then the condition in (3.4) becomes

$$
1+\frac{\mathcal{C}_{1} \cdot \mathcal{C}_{2}}{\mathcal{C}_{1} \mathcal{C}_{2}}<0
$$

The general principle of defining $\eta_{2}$ in (3.9) can be straightforwardly understood as follows. By writing the Möbius transformations in Eqs. (2.9) and (2.10) in terms of Pauli matrices, one can find that in the limit $\frac{T_{i}}{l} \rightarrow \infty$ $(i=1,2)$ the sum of coefficients of the leading terms in $\operatorname{Tr}\left(M_{1} \cdot M_{2}\right)$ is nothing but $\eta_{2}$. If $\eta_{2}<0$, then one has $\operatorname{Tr}\left(M_{1} \cdot M_{2}\right)=-\infty$ in the limit $\frac{T_{i}}{l} \rightarrow \infty(i=1,2)$. In the other limit $\frac{T_{i}}{l} \rightarrow 0(i=1,2)$, one can find that $\operatorname{Tr}\left(M_{1} \cdot M_{2}\right) \rightarrow 2$. Therefore, as we change the value of $\frac{T_{i}}{l}$ continuously, there must be a non-heating phase with $\left|\operatorname{Tr}\left(M_{1} \cdot M_{2}\right)\right|<2$.

Now we give an illustration based on the hyperbolichyperbolic driving. One can find details for other cases in Appendix B. In the hyperbolic-hyperbolic driving, one can find that

$$
\begin{aligned}
& \operatorname{Tr}\left(M_{1} \cdot M_{2}\right)=2 \cosh \left(\frac{\pi \mathcal{C}_{1} T_{1}}{l}-\frac{\pi \mathcal{C}_{2} T_{2}}{l}\right) \\
& +2\left(1+\frac{\mathcal{C}_{1} \cdot \mathcal{C}_{2}}{\mathcal{C}_{1} \cdot \mathcal{C}_{2}}\right) \cdot \sinh \left(\frac{\pi \mathcal{C}_{1} T_{1}}{l}\right) \cdot \sinh \left(\frac{\pi \mathcal{C}_{2} T_{2}}{l}\right) .
\end{aligned}
$$

For $1+\frac{\mathcal{C}_{1} \cdot \mathcal{C}_{2}}{\mathcal{C}_{1} \cdot \mathcal{C}_{2}}=0$, the Floquet CFT will stay at the phase transition (or critical phase) along the line $\frac{\mathcal{C}_{1} T_{1}}{l}=\frac{\mathcal{C}_{2} T_{2}}{l}$. Away from this critical line, the driven CFT will always be in the heating phase. For $1+\frac{\mathcal{C}_{1} \cdot \mathcal{C}_{1}}{\mathcal{C}_{1} \cdot \mathcal{C}_{2}}>0$, one always has $\operatorname{Tr}\left(M_{1} \cdot M_{2}\right)>2$ for arbitrary $0<T_{1} / l, T_{2} / l<\infty$, and therefore the system is always in the heating phase. The non-heating phase can appear if and only if (3.11) is satisfied, which can be understood as follows. In the limit $T_{1} / l, T_{2} / l \rightarrow 0$, one has $\operatorname{Tr}\left(M_{1} \cdot M_{2}\right) \rightarrow 2$. In the other limit $T_{1} / l, T_{2} / l \rightarrow \infty$, one has $\operatorname{Tr}\left(M_{1} \cdot M_{2}\right) \rightarrow-\infty$. By continuously changing $T_{1} / l$ and $T_{2} / l$, there must exist a region where $\left|\operatorname{Tr}\left(M_{1} \cdot M_{2}\right)\right|<2$, which corresponds to the non-heating phase. As an intuitive picture, by fixing $\frac{\mathcal{C}_{1}}{\mathcal{C}_{1}}=\left(\sigma_{1}^{0}, \sigma_{1}^{+}, \sigma_{1}^{-}\right)=(0,1,0)$, the vector $\frac{\mathcal{C}_{2}}{\mathcal{C}_{2}}$ that satisfies (3.11) is shown in Fig. 4.

Before we leave this subsection, we hope to point out one interesting feature in the phase diagram of hyperbolic-hyberbolic driven Floquet CFT. As we approach $1+\frac{\mathcal{C}_{1} \cdot \mathcal{C}_{2}}{\mathcal{C}_{1} \cdot \mathcal{C}_{2}}=0$ from $(3.11)$, it is found that the non-heating phase does not vanish continuously. What we observe is that the non-heating phase is composed of an island connected to three lines (see Fig. 5). As we approach $1+\frac{\mathcal{C}_{1} \cdot \mathcal{C}_{2}}{\mathcal{C}_{1} \cdot \mathcal{C}_{2}}=0$, this island of non-heating phase does not vanish but simply moves to the infinity (See Appendix B for more details).

\section{C. $\quad N=3$}

Now let us consider the case of $N=3$, i.e., there are three driving Hamiltonians $H_{1}, H_{2}$, and $H_{3}$ in a driving 

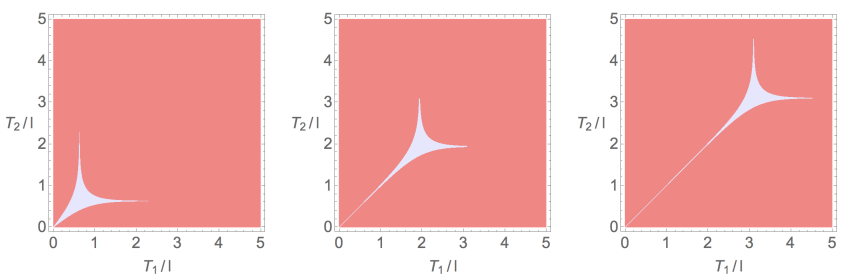

FIG. 5. Phase diagram in a Floquet CFT with $N=2$ driving Hamiltonians, both of which are of hyperbolic types. The corresponding Casimir vectors are $\mathcal{C}_{1}=(1, a, 0)$ and $\mathcal{C}_{2}=$ $(1,0, a)$, where we choose $a=1.4$ (left), 1.41421 (middle), and 1.41421356 (right). The location of the non-heating phase in blue will move to infinity as we approach $a=\sqrt{2}$ from $a<\sqrt{2}$. For $a>\sqrt{2}$, the condition in (3.11) is violated, and the non-heating phase does not exist.

period. Similar to the previous subsection, we determine the phase diagram based on the value of $\left|\operatorname{Tr}\left(M_{1} \cdot M_{2} \cdot M_{3}\right)\right|$ according to the criteria in (2.14).

One can find there are in general three layers of conditions to ensure the existence of non-heating phases:

1. Quasi- $n$ (with $n=1$ ) condition. In this case, one simply needs to look at if there is an elliptic Hamiltonian in the driving. If there is, then there must exist a non-heating phase in the phase diagram. In particular, the non-heating phase can be realized when the elliptic Hamiltonian dominates in the driving.

2. Quasi- $n$ (with $n=2$ ) condition. One needs to consider all possible choices of pairings $(i, j)$ in the driving. If both Hamiltonians are non-elliptic, then the quasi- $n(n=2)$ condition is

$$
\eta_{n=2}<0
$$

One can find that these conditions are nothing but those obtained in Table I. In particular, the nonheating phases are realized when $H_{i}$ and $H_{j}$ dominate in the driving.

3. Intrinsic- $N(N=3)$ condition. Now we need to consider all the three driving Hamiltonians together. If all the three Hamiltonians are nonelliptic, then the intrinsic- $N(N=3)$ condition ensuring the existence of non-heating phases is

$$
\eta_{N=3}<0,
$$

where we have defined

$$
\eta_{N=3}:=\operatorname{Tr}\left(P_{1} \cdot P_{2} \cdot P_{3}\right),
$$

with the matrices $P_{j}(j=1,2,3)$ of the form in Eqs. (3.5) or (3.6) depending on the types of Hamiltonian $H_{j}$.
If at least one the above conditions is satisfied, then there must exist a non-heating phase.

The condition in (3.14) is obtained in a similar way to that in obtaining (3.4). That is, by tracking the behavior of $\operatorname{Tr}\left(M_{1} \cdot M_{2} \cdot M_{3}\right)$ with $\frac{T_{i}}{l}(i=1,2,3)$ varied from $0^{+}$ to $\infty$, one can find that the condition $\eta_{N=3}<0$ ensures the existence of non-heating phases.

Let us consider examples on the expression of $\eta_{N=3}$ in (3.15) (One can find more examples in Appendix C). If all the three Hamiltonians are parabolic, one has

$$
\eta_{N=3}=\mathcal{C}_{1} * \mathcal{C}_{2} * \mathcal{C}_{3},
$$

where we have defined

$$
\begin{aligned}
\mathcal{C}_{1} * \mathcal{C}_{2} * \mathcal{C}_{3}:= & \sigma_{1}^{0} \sigma_{2}^{+} \sigma_{3}^{-}-\sigma_{1}^{0} \sigma_{2}^{-} \sigma_{3}^{+}+\sigma_{1}^{+} \sigma_{2}^{-} \sigma_{3}^{0} \\
& -\sigma_{1}^{+} \sigma_{2}^{0} \sigma_{3}^{-}+\sigma_{1}^{-} \sigma_{2}^{0} \sigma_{3}^{+}-\sigma_{1}^{-} \sigma_{2}^{+} \sigma_{3}^{0} .
\end{aligned}
$$

If all the three Hamiltonians are of hyperbolic types, then $\eta$ can be expressed as

$$
\eta_{N=3}=1+\sum_{i<j} \frac{\mathcal{C}_{i} \cdot \mathcal{C}_{j}}{\mathcal{C}_{i} \mathcal{C}_{j}}+\frac{\mathcal{C}_{1} * \mathcal{C}_{2} * \mathcal{C}_{3}}{\mathcal{C}_{1} \mathcal{C}_{2} \mathcal{C}_{3}}
$$

Next, let us illustrate the intrinsic- $N(N=3)$ conditions in (3.14) explicitly with these two specific cases.

In the first illustrating case, all the three driving Hamiltonians are parabolic. Based on the Möbius transformation in (2.9), one can obtain

$$
\begin{aligned}
& \operatorname{Tr}\left(M_{1} \cdot M_{2} \cdot M_{3}\right)=2\left(1+\sum_{i<j}^{3} \mathcal{C}_{i} \cdot \mathcal{C}_{j} \frac{\pi T_{i}}{l} \cdot \frac{\pi T_{j}}{l}\right. \\
& \left.+\frac{\pi T_{1}}{l} \cdot \frac{\pi T_{2}}{l} \cdot \frac{\pi T_{3}}{l} \mathcal{C}_{1} * \mathcal{C}_{2} * \mathcal{C}_{3}\right) .
\end{aligned}
$$

For $\frac{\pi T_{i}}{l} \rightarrow 0^{+}(i=1,2,3)$, one has $\operatorname{Tr}\left(M_{1} \cdot M_{2} \cdot M_{3}\right) \rightarrow 2$. In the other limit $\frac{\pi T_{i}}{l} \rightarrow \infty$, the last term in (3.19) will dominate. If $\mathcal{C}_{1} * \mathcal{C}_{2} * \mathcal{C}_{3}<0$, then we will have $\operatorname{Tr}\left(M_{1}\right.$. $\left.M_{2} \cdot M_{3}\right) \rightarrow-\infty$. That is, as we increase $\frac{\pi T_{i}}{l}, \operatorname{Tr}\left(M_{1} \cdot M_{2}\right.$. $M_{3}$ ) changes from 2 to $-\infty$ continuously. Apparently, there will be a non-heating phase (with $\mid \operatorname{Tr}\left(M_{1} \cdot M_{2}\right.$. $\left.M_{3}\right) \mid<2$ ) in the parameter space.

In the second illustrating case, we consider three hyperbolic Hamiltonians. Based on the Möbius transformations in (2.10), one can find that

$$
\begin{aligned}
& \operatorname{Tr}\left(M_{1} \cdot M_{2} \cdot M_{3}\right)=2\left[\cosh \frac{\pi \mathcal{C}_{1} T_{1}}{l} \cosh \frac{\pi \mathcal{C}_{2} T_{2}}{l} \cosh \frac{\pi \mathcal{C}_{3} T_{3}}{l}\right. \\
& +\sum_{i<j ; k \neq i, j}^{3} \frac{\mathcal{C}_{i} \cdot \mathcal{C}_{j}}{\mathcal{C}_{i} \mathcal{C}_{j}} \sinh \frac{\pi \mathcal{C}_{i} T_{i}}{l} \sinh \frac{\pi \mathcal{C}_{j} T_{j}}{l} \cosh \frac{\pi \mathcal{C}_{k} T_{k}}{l} \\
& \left.+\frac{\mathcal{C}_{1} * \mathcal{C}_{2} * \mathcal{C}_{3}}{\mathcal{C}_{1} \mathcal{C}_{2} \mathcal{C}_{3}} \sinh \frac{\pi \mathcal{C}_{1} T_{1}}{l} \sinh \frac{\pi \mathcal{C}_{2} T_{2}}{l} \sinh \frac{\pi \mathcal{C}_{3} T_{3}}{l}\right] .
\end{aligned}
$$

As before, by choosing $\frac{\mathcal{C}_{i} T_{i}}{l} \rightarrow 0^{+}(i=1,2,3)$, one has $\operatorname{Tr}\left(M_{1} \cdot M_{2} \cdot M_{3}\right) \simeq 2$. Next, by taking the limit $\frac{\mathcal{C}_{i} T_{i}}{l} \rightarrow \infty$, one has $\cosh \frac{\pi \mathcal{C}_{i} T_{i}}{l} \simeq \sinh \frac{\pi \mathcal{C}_{i} T_{i}}{l}$, and therefore $\operatorname{Tr}\left(M_{1} \cdot M_{2}\right.$. $\left.M_{3}\right) \simeq-2 \eta_{N=3}\left(\cosh \frac{\pi \mathcal{C}_{1} T_{1}}{L} \cosh \frac{\pi \mathcal{C}_{2} T_{2}}{l} \cosh \frac{\pi \mathcal{C}_{3} T_{3}}{l}\right)$, where 

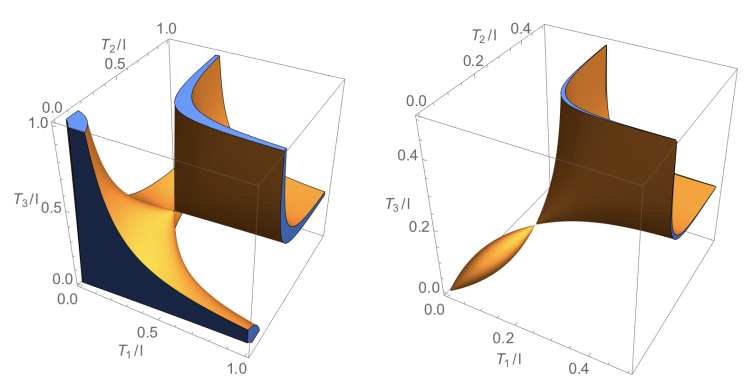

FIG. 6. Non-heating phases in a Floquet CFT with $N=3$ driving Hamiltonians, all of which are of hyperbolic types. We choose $\lambda=1.1$ (left) in (3.21) such that only the condition $\eta_{n=2}<0$ is satisfied, and $\lambda=3$ (right) such that only the condition $\eta_{N=3}<0$ is satisfied. The complemented regions are in the heating phase.

$\eta_{N=3}$ is defined in Eq. (3.18). If $\eta_{N=3}<0$, we will have $\operatorname{Tr}\left(M_{1} \cdot M_{2} \cdot M_{3}\right) \rightarrow-\infty$. Therefore, as we tune $\frac{\mathcal{C}_{i} T_{i}}{l}$ from $0^{+}$to $\infty$, the amplitude of $\operatorname{Tr}\left(M_{1} \cdot M_{2} \cdot M_{3}\right)$ will change from -2 to $\infty$ continuously. Apparently, there will be a non-heating phase (with $\left|\operatorname{Tr}\left(M_{1} \cdot M_{2} \cdot M_{3}\right)\right|<2$ ) in the parameter space.

To have an intuitive picture of the phase diagram, we give a sample plot of the non-heating phases when the driving Hamiltonians are all hyperbolic. We consider the Casimir vectors:

$$
\mathcal{C}_{i}=\left(1, \lambda \cos \theta_{i}, \lambda \sin \theta_{i}\right),
$$

where $\lambda>1$ such that the deformed Hamiltonian is always of hyperbolic type. By choosing $\theta_{1}=0, \theta_{2}=\frac{2 \pi}{3}$, and $\theta_{3}=\frac{4 \pi}{3}$, one can check explicitly that for $i, j \in 1,2,3$ and $i \neq j$, one always has

$$
\eta_{2}=\frac{1}{2}-\frac{3}{2} \frac{1}{\lambda^{2}-1}
$$

and

$$
\eta_{3}=-\frac{1}{2}-\frac{9}{2} \cdot \frac{1}{\lambda^{2}-1}+\frac{3 \sqrt{3}}{2} \cdot \frac{\lambda^{2}}{\left(\lambda^{2}-1\right)^{3 / 2}} .
$$

Then by solving the conditions in (3.13) and (3.14), one can obtain $\eta_{2}<0$ when $1<\lambda<2$, and $\eta_{3}<0$ when $\lambda>2$. Typical plots of the phase diagrams for these two cases can be found in Fig. 6 .

\section{General $N$}

Based on the discussions in the previous subsections, now we are ready to give the conditions for the existence of non-heating phases in an $\mathrm{SL}_{2}$ deformed Floquet $\mathrm{CFT}$ when there are $N$ driving Hamiltonians.

Let us denote the $N$ driving Hamiltonians as $\left\{H_{1}, H_{2}, \cdots, H_{N}\right\}$, which are arranged in time order of driving. That is, we drive the CFT with $H_{1}$ for time duration $T_{1}, H_{2}$ for time duration $T_{2}$, and so on. These driving Hamiltonians are characterized by Casimir vectors $\left\{\mathcal{C}_{1}, \mathcal{C}_{2}, \cdots, \mathcal{C}_{N}\right\}$ as defined in (3.2).

The sufficient conditions for the existence of nonheating phases are composed of $N$ layers of conditions, which add constraints on the Casimir vectors $\left\{\mathcal{C}_{1}, \mathcal{C}_{2}, \cdots, \mathcal{C}_{N}\right\}$. In layer $n(1 \leq n \leq N)$, we consider all possible choices of sets $\left\{H_{i_{1}}, \cdots, H_{i_{n}}\right\}$, where the Hamiltonians are again arranged in the time order of driving. Then the layer- $n$ conditions are:

1. If there is at least one elliptic Hamiltonian in the set $\left\{H_{i_{1}}, \cdots, H_{i_{n}}\right\}$, then there is no constraint on the Casimir vectors $\left\{\mathcal{C}_{i_{1}}, \mathcal{C}_{i_{2}}, \cdots, \mathcal{C}_{i_{n}}\right\}$.

2. If all the Hamiltonians in $\left\{H_{i_{1}}, \cdots, H_{i_{n}}\right\}$ are nonelliptic (either parabolic or hyperbolic), then the conditions ensuring the existence of non-heating phases are

$$
\exists \eta_{n}<0, \quad n=1, \cdots, N .
$$

Here the indicator $\eta_{n}$ is defined as

$$
\eta_{n}:=\operatorname{Tr}\left(P_{i_{1}} \cdot P_{i_{2}} \cdots P_{i_{n}}\right),
$$

with each matrix $P_{j}$ determined by the Casimir vectors $\mathcal{C}_{j}=\left(\sigma_{j}^{0}, \sigma_{j}^{+}, \sigma_{j}^{-}\right)$as follows. If the driving Hamiltonian $H_{j}$ is parabolic, then $P_{j}$ has the form

$$
P_{j}=\left(\begin{array}{cc}
i \sigma_{j}^{0} & i\left(\sigma_{j}^{+}+i \sigma_{j}^{-}\right) \\
-i\left(\sigma_{j}^{+}-i \sigma_{j}^{-}\right) & -i \sigma_{j}^{0}
\end{array}\right) .
$$

If the driving Hamiltonian $H_{j}$ is hyperbolic, then $P_{j}$ has the form

$$
P_{j}=\left(\begin{array}{cc}
1+i \frac{\sigma_{j}^{0}}{\mathcal{C}_{j}} & i \frac{\left(\sigma_{j}^{+}+i \sigma_{j}^{-}\right)}{\mathcal{C}_{j}} \\
-i \frac{\left(\sigma_{j}^{+}-i \sigma_{j}^{-}\right)}{\mathcal{C}_{j}} & 1-i \frac{\sigma_{j}^{0}}{\mathcal{C}_{j}}
\end{array}\right) .
$$

By considering all possible $1 \leq n \leq N$, there are in total $2^{N}-1$ conditions. If at least one of these conditions is satisfied, then there must exist non-heating phases in the phase diagram.

Now we give several remarks on the layer- $n$ conditions:

- The way to obtain condition (3.24) is similar to the examples considered in Sec. III B and IIIC. That is, $\eta_{n}$ corresponds to the sum of coefficients of the leading-order terms in $\operatorname{Tr}\left(M_{i_{1}} \cdot M_{i_{2}} \cdots M_{i_{n}}\right)$ in the limit $T_{i_{k}} / l \rightarrow \infty$ for all $i_{k} \in\left\{i_{1}, i_{2}, \cdots, i_{n}\right\}$. Then the condition in (3.24) ensures that $\operatorname{Tr}\left(M_{i_{1}} \cdot M_{i_{2}} \cdots M_{i_{n}}\right)$ will change from 2 to $-\infty$ as we tune $T_{i_{k}} / l$ from 0 to $\infty$ continuously. Then there must exist non-heating phases with $\left|\operatorname{Tr}\left(M_{i_{1}} \cdot M_{i_{2}} \cdots M_{i_{n}}\right)\right|<2$.

- It is noted that there are in total $C_{N}^{n}=\frac{N !}{(N-n) ! n !}$ conditions in layer- $n$ conditions. In addition, as we have mentioned, if there exists at least one elliptic Hamiltonian in the chosen set $\left\{H_{i_{1}}, \cdots, H_{i_{n}}\right\}$, there is no constraint on the corresponding Casimir vectors $\left\{\mathcal{C}_{i_{1}}, \mathcal{C}_{i_{2}}, \cdots, \mathcal{C}_{i_{n}}\right\}$. We hope to emphasize that this does not mean there is no 
constraint on the layer- $n^{\prime}$ condition when $n^{\prime}<n$, because it is totally possible there is no elliptic Hamiltonian in the subset $\left\{H_{i_{1}}, \cdots, H_{i_{n^{\prime}}}\right\} \subset\left\{H_{i_{1}}, \cdots, H_{i_{n}}\right\}$.

- In the specific case $n=1$, the layer- $n$ condition mentioned above is simply reduced to the existence of an elliptic driving Hamiltonian.

Finally, let us comment on where to find these nonheating phases in the $N$-dimensional parameter space spanned by $\left\{\frac{T_{1}}{l}, \frac{T_{2}}{l}, \cdots, \frac{T_{N}}{l}\right\}$. Suppose a certain layer- $n$ condition is satisfied, then if there exists at least one elliptic Hamiltonian (which we denote as $H_{i_{m}}$ ) in the subset $\left\{H_{i_{1}}, \cdots, H_{i_{n}}\right\}$, then the non-heating phase can be obtained by taking all $T_{i} / l \rightarrow 0$ but with a finite $T_{i_{m}} / l$; if all the $n$ Hamiltonians in $\left\{H_{i_{1}}, \cdots, H_{i_{n}}\right\}$ are nonelliptic, then the non-heating phases can be found in the $n$-dimensional subspace spanned by $\left\{\frac{T_{i_{1}}}{l}, \frac{T_{i_{2}}}{l}, \cdots, \frac{T_{i_{n}}}{l}\right\}$. One can simply take $\frac{T_{i_{1}}}{l}=\frac{T_{i_{2}}}{l}=\cdots=\frac{T_{i_{n}}}{l}:=\frac{T^{*}}{l}$. By increasing $\frac{T^{*}}{l}$ from 0 to $\infty$ gradually, one will necessarily find a non-heating phase. For example, one can refer to Fig. 5 for the case of $N=n=2$ and the right plot in Fig. 6 for the case of $N=n=3$.

\section{CONCLUSION AND DISCUSSION}

In this paper, we have studied how the types of driving Hamtiltonians affect the phase diagrams in an $\mathrm{SL}_{2}$ deformed Floquet CFT. It is found that the heating phases are generic, but the non-heating phases may be absent in the phase diagram. We give the $N$-layer conditions (with each layer of conditions expressed in (3.24)) for the existence of non-heating phases in an $\mathrm{SL}_{2}$ deformed Floquet CFT with $N$ driving Hamiltonians. We showed that these conditions are sufficient and necessary for $N=2$. For $N>2$, we only showed that these conditions are sufficient. In fact, for small $N$ with $N>2$, we have scanned the parameter space numerically and did not find any non-heating phases if the conditions in (3.24) are violated. We conjecture that our conditions in (3.24) are also necessary conditions. It is an interesting future problem to prove this conjecture.

Besides the types of driving Hamiltonians, the concrete driving sequences will also affect the phase diagram, such as quasi-periodic drivings and random drivings, as discussed in Refs. 55-57. One simple form of the quasiperiodic drivings is the Fibonacci quasi-periodic driving as studied in Refs. 55 and 56 recently. One way to obtain the phase diagram is to use a periodic driving to approach the quasi-periodic driving, by taking larger and larger driving period. Our conditions may be helpful to understand how the phase diagram in a quasi-periodically driven CFT depends on the types of driving Hamiltonians. In the random drivings, the dependence of phase diagrams on the types of driving Hamiltonians will also exhibit very interesting structures, as will be discussed in detail in Ref. 57.
One interesting problem is to generalize the $\mathrm{SL}_{2}$ deformations to the more general deformations, by choosing a general real function $f(x)$ in (1.2), where the underlying group structure is the Virasoro group. Recently, in Ref. 64, the authors consider related problems in an opposite way. That is, one can start from a certain interesting conformal map on the complex $z$-plane, and map it back to the physical spacetime to find out the corresponding deformation of the energy-momentum tensor. In general, this 'mapping back' procedure cannot be analytically done, and one needs to perform numerical calculations. In addition, we hope to emphasize that it is possible that the envelope function $f(x)$ [see Eq. (1.2)] generated in this way may be not a real function, which may result in non-Hermitian deformed Hamiltonians. Nevertheless, one may use this method to search for interesting conformal maps under which the driven CFT exhibit exotic features.

Another interesting problem is on the characterization of the Floquet CFTs. Previous works characterize the phase diagrams based on either entanglement entropy or energy evolution. ${ }^{39,53-57}$ More detailed features of the time-dependent driven CFT can be captured by the entanglement Hamiltonian (and its spectrum), which was recently used to study the non-equilibrium dynamics such as quantum quenches in $(1+1) \mathrm{d}$ CFTs both analytically ${ }^{65,66}$ and numerically ${ }^{67,68}$. In the setup of Floquet CFTs with $\mathrm{SL}_{2}$ deformation, we expect that the entanglement Hamiltonians in different phases of Floquet CFTs may be classified into three types (see (1.6)) up to certain envelope functions. We will leave this problem to a future work.

\section{ACKNOWLEDGMENTS}

We thank B. Beri, R. Fan, Y. Gu, H. Shapourian, C. von Keyserlingk, A. Ludwig, I. Martin, S. Ryu, T. Tada, A. Vishwanath, A. Wall and J. Q. Wu for useful discussions. X. W. also thanks D. Ageev, A. Bagrov, and A. Iliasov for communications on Ref. 64. B. H. is supported by ERC Starting Grant No. 678795 TopInSy. $\mathrm{X}$. W. is supported by Gordon and Betty Moore Foundation's EPiQS initiative through Grant No. GBMF4303 at MIT.

\section{Appendix A: $N=1$}

In this appendix, we give further detailed discussions on the time evolution of entanglement entropy after a single quantum quench. Let us first consider the simple choice of subsystem $A=[n l,(n+1) l]$ where $l=L / n$. Let us keep the anti-chiral part undeformed, and only focus on the effect of deformation in the chiral part. Then based on Eq. (2.17) and Eqs. (2.8), (2.9), and (2.10), one can obtain 


$$
S_{A}(t)-S_{A}(0)= \begin{cases}\frac{c}{6} \log \left\{\left[\cosh \left(\frac{\pi \mathcal{C} t}{l}\right)-\frac{\sigma^{-}}{\mathcal{C}} \sinh \left(\frac{\pi \mathcal{C} t}{l}\right)\right]^{2}+\left[\frac{\sigma^{0}+\sigma^{+}}{\mathcal{C}} \cdot \sinh \left(\frac{\pi \mathcal{C} t}{l}\right)\right]^{2}\right\}, & c^{(2)}>0 \\ \frac{c}{6} \log \left\{\left(1-\frac{\pi \sigma^{-} t}{l}\right)^{2}+\left(\frac{\pi\left(\sigma^{0}+\sigma^{+}\right) t}{l}\right)^{2}\right\} & c^{(2)}=0 \\ \frac{c}{6} \log \left\{\left[\cos \left(\frac{\pi \mathcal{C} t}{l}\right)-\frac{\sigma^{-}}{\mathcal{C}} \sin \left(\frac{\pi \mathcal{C} t}{l}\right)\right]^{2}+\left[\frac{\sigma^{0}+\sigma^{+}}{\mathcal{C}} \cdot \sin \left(\frac{\pi \mathcal{C} t}{l}\right)\right]^{2}\right\}, & c^{(2)}<0\end{cases}
$$

For general choices of $\left(\sigma^{0}, \sigma^{+}, \sigma^{-}\right)$, one can find that in the long time driving limit $t / l \gg 1$, the entanglement entropy can be approximated by the formulas in (3.1) in the main text. But there is one subtlety we hope to point out. In the case of $c^{(2)}>0$, one can find that by choosing $\sigma^{-} \neq 0$ and $\sigma^{0}=\sigma^{+}=0$, one has

$$
S_{A}(t)-S_{A}(0)=-\frac{c}{6} \cdot \frac{\pi \mathcal{C} t}{l}
$$

That is, the entanglement entropy decreases linearly in time. This phenomenon has been analyzed in Ref. 55 . The reason is that the entanglement cut and the energymomentum density peaks coincide with each other. Intuitively, in the study of $S_{A}(t)$, one needs to introduce a UV cutoff at the entanglement cuts. Since the energymomentum density peaks are also located at the entanglement cuts, during the driving, the degree of freedom that carries the entanglement between $A$ and its complement will accumulate at the entanglement cut. Due to the UV cut-off, these degrees of freedom cannot be detected by the entanglement entropy, which results in a decrease in the entanglement entropy. To see the linear growth of the entanglement entropy in this case, one simply needs to shift the locations of entanglement cuts. For example, by choosing $A=\left[\left(k+\frac{1}{2}\right) l,\left(k+\frac{3}{2}\right) l\right]$ where $k \in \mathbb{Z}$, the entanglement entropy is expressed in (2.19). In this case, the entanglement cuts and energy-momentum density peaks do not coincide with each other. With the same choice of $\sigma^{-} \neq 0$ and $\sigma^{0}=\sigma^{+}=0$, one can find that the entanglement entropy grows linearly in time now.

\section{Appendix B: $N=2$}

In this appendix, we give a derivation of the results in Table. I in the main text. That is, we consider six different pairings of $H_{1}$ and $H_{2}$ with the Hamiltonian types in (1.6): (i) elliptic-elliptic, (ii) elliptic-parabolic, (iii) elliptic-hyperbolic, (iv) parabolicparabolic, (v) parabolic-hyperbolic, and (vi) hyperbolichyperbolic. Some detailed features of the phase diagram will also be discussed.

\section{Features of phase diagram}

\section{(i) Elliptic-elliptic}

If both the driving Hamiltonians are elliptic, then based on the Möbius transformations in Eqs. (2.8), (2.9), (2.10), we have

$$
\begin{array}{r}
\operatorname{Tr}\left(M_{1} \cdot M_{2}\right)=2 \cos \left(\frac{\pi \mathcal{C}_{1} T_{1}}{l}\right) \cdot \cos \left(\frac{\pi \mathcal{C}_{2} T_{2}}{l}\right) \\
\quad+\frac{2 \mathcal{C}_{1} \cdot \mathcal{C}_{2}}{\mathcal{C}_{1} \cdot \mathcal{C}_{2}} \cdot \sin \left(\frac{\pi \mathcal{C}_{1} T_{1}}{l}\right) \cdot \sin \left(\frac{\pi \mathcal{C}_{2} T_{2}}{l}\right) .
\end{array}
$$

First, as proved in Appendix B 2, there always exists a heating phase along the lines in Eqs. (B18) and (B19) in the parameter space.

Second, let us prove there always exist non-heating phases in the phase diagram. It is noted that when both $H_{1}$ are $H_{2}$ are elliptic, we always have $\left|\frac{\mathcal{C}_{1} \cdot \mathcal{C}_{2}}{\mathcal{C}_{1} \mathcal{C}_{2}}\right|>1$, as discussed in Appendix B 2. Let us consider the cases with $\frac{\mathcal{C}_{1} \cdot \mathcal{C}_{2}}{\mathcal{C}_{1} \mathcal{C}_{2}}<-1$ and $\frac{\mathcal{C}_{1} \cdot \mathcal{C}_{2}}{\mathcal{C}_{1} \mathcal{C}_{2}}>-1$ separately.

For $\frac{\mathcal{C}_{1} \cdot \mathcal{C}_{2}}{\mathcal{C}_{1} \mathcal{C}_{2}}<-1$, it is convenient to rewrite Eq.(B1) as follows

$$
\begin{aligned}
\operatorname{Tr}\left(M_{1} \cdot M_{2}\right)= & 2 \cos \left(\frac{\pi \mathcal{C}_{1} T_{1}}{l}+\frac{\pi \mathcal{C}_{2} T_{2}}{l}\right) \\
& +2\left(\frac{\mathcal{C}_{1} \cdot \mathcal{C}_{2}}{\mathcal{C}_{1} \mathcal{C}_{2}}+1\right) \cdot \sin \left(\frac{\pi \mathcal{C}_{1} T_{1}}{l}\right) \cdot \sin \left(\frac{\pi \mathcal{C}_{2} T_{2}}{l}\right) .
\end{aligned}
$$

One can find that for $\frac{\mathcal{C}_{1} T_{1}}{l} \simeq 0^{+}$and $\frac{\mathcal{C}_{2} T_{2}}{l} \simeq 0^{+}$, one has $0<\operatorname{Tr}\left(M_{1} \cdot M_{2}\right)<2$, and therefore the system is in a non-heating phase.

Similarly, for $\frac{\mathcal{C}_{1} \cdot \mathcal{C}_{2}}{\mathcal{C}_{1} \mathcal{C}_{2}}>1$, one can rewrite Eq.(B1) as

$$
\begin{aligned}
\operatorname{Tr}\left(M_{1} \cdot M_{2}\right)= & 2 \cos \left(\frac{\pi \mathcal{C}_{1} T_{1}}{l}-\frac{\pi \mathcal{C}_{2} T_{2}}{l}\right) \\
& +2\left(\frac{\mathcal{C}_{1} \cdot \mathcal{C}_{2}}{\mathcal{C}_{1} \mathcal{C}_{2}}-1\right) \cdot \sin \left(\frac{\pi \mathcal{C}_{1} T_{1}}{l}\right) \cdot \sin \left(\frac{\pi \mathcal{C}_{2} T_{2}}{l}\right) .
\end{aligned}
$$

For $\frac{\mathcal{C}_{1} T_{1}}{l} \simeq 0^{+}$and $\frac{\mathcal{C}_{2} T_{2}}{l} \simeq 1-0^{+}$, or $\frac{\mathcal{C}_{1} T_{1}}{l} \simeq 1-0^{+}$and $\frac{\mathcal{C}_{2} T_{2}}{l} \simeq 0^{+}$, we have $-2<\operatorname{Tr}\left(M_{1} \cdot M_{2}\right)<0$, and therefore the system is in a non-heating phase.

For the two regions corresponding to non-heating phases as discussed above, one can see Fig. 8 for example.

In short, when the two non-commuting driving Hamiltonians are both elliptic, there are both heating and nonheating phases in the phase diagram.

(ii) Elliptic-parabolic

Without loss of generality, we consider the case that $H_{1}$ is elliptic, and $H_{2}$ is parabolic. Then we have

$$
\begin{aligned}
\operatorname{Tr}\left(M_{1} \cdot M_{2}\right)= & 2 \cos \left(\frac{\pi \mathcal{C}_{1} T_{1}}{l}\right) \\
& +\frac{2 \mathcal{C}_{1} \cdot \mathcal{C}_{2}}{\mathcal{C}_{1}} \cdot \frac{\pi T_{2}}{l} \cdot \sin \left(\frac{\pi \mathcal{C}_{1} T_{1}}{l}\right)
\end{aligned}
$$


where $\mathcal{C}_{1} \cdot \mathcal{C}_{2} \neq 0$. For $\mathcal{C}_{1} \cdot \mathcal{C}_{2}<0$, one can find that for $\frac{\pi \mathcal{C}_{1} T_{1}}{l} \simeq 0^{+}$and $T_{2} / l \simeq 0^{+}$, we always have $0<$ $\operatorname{Tr}\left(M_{1} \cdot M_{2}\right)<2$, and therefore the system is in a nonheating phase. On the other hand, for finite $\frac{\pi \mathcal{C}_{1} T_{1}}{l}$, as $T_{2}$ goes to infinity, we always have a heating phase. For $\mathcal{C}_{1} \cdot \mathcal{C}_{2}>0$, one can find that for $\frac{\pi \mathcal{C}_{1} T_{1}}{l} \simeq 1-0^{+}$and $\frac{\pi \mathcal{C}_{2} T_{2}}{l} \simeq 0^{+}$, we always have $0<\operatorname{Tr}\left(M_{1} \cdot M_{2}\right)<2$, and therefore the system is in a non-heating phase.

(iii) Elliptic-hyperbolic

Without loss of generality, we consider the case that $H_{1}$ is elliptic, and $H_{2}$ is hyperbolic. Then we have

$$
\begin{aligned}
\operatorname{Tr}\left(M_{1} \cdot M_{2}\right)= & 2 \cos \left(\frac{\pi \mathcal{C}_{1} T_{1}}{l}\right) \cdot \cosh \left(\frac{\pi \mathcal{C}_{2} T_{2}}{l}\right) \\
& +\frac{2 \mathcal{C}_{1} \cdot \mathcal{C}_{1}}{\mathcal{C}_{1} \cdot \mathcal{C}_{2}} \cdot \sin \left(\frac{\pi \mathcal{C}_{1} T_{1}}{l}\right) \cdot \sinh \left(\frac{\pi \mathcal{C}_{2} T_{2}}{l}\right) .
\end{aligned}
$$

For $\mathcal{C}_{1} \cdot \mathcal{C}_{2}=0$, it is straightforward to check that both the heating and non-heating phases can exist in the phase diagram. For example, the driven CFT is always in the non-heating phase along the lines $\frac{\mathcal{C}_{1} T_{1}}{l}=\frac{1}{2}+n$, where $n \in \mathbb{Z}$ (See Fig. 3). If $\frac{\mathcal{C}_{1} T_{1}}{l} \neq \frac{1}{2}+n$, the driven CFT will be in the heating phase for large enough $\frac{T_{2}}{l}$. For $\mathcal{C}_{1} \cdot \mathcal{C}_{2} \neq 0$, one can always write Eq. (B5) in the form of $N \cos \left(\frac{\pi \mathcal{C}_{1} T_{1}}{l}+\phi\right)$, where $|N|$ increases exponentially with $\mathcal{C}_{2} T_{2} / l$ for $\mathcal{C}_{2} T_{2} / l \gg 1$. Then the system is in the non-heating phase along the lines $\frac{\pi \mathcal{C}_{1} T_{1}}{l}+\phi=(1 / 2+n) \pi$ where $n \in \mathbb{Z}$, and in the heating phase when $\frac{\pi \mathcal{C}_{1} T_{1}}{l}+$ $\phi \neq(1 / 2+n) \pi$ and $\frac{\mathcal{C}_{2} T_{2}}{l}$ is large enough. Therefore, for arbitrary $\mathcal{C}_{1} \cdot \mathcal{C}_{2}$, we always have both heating and non-heating phases in the phase diagram.

(iv) Parabolic-parabolic

If both the driving Hamiltonians are parabolic, we have

$$
\operatorname{Tr}\left(M_{1} \cdot M_{2}\right)=2+2 \mathcal{C}_{1} \cdot \mathcal{C}_{2} \cdot \frac{\pi T_{1}}{l} \cdot \frac{\pi T_{2}}{l},
$$

where $\mathcal{C}_{1} \cdot \mathcal{C}_{2} \neq 0$. Since $T_{1}, T_{2}>0$, one can find that there are both heating and non-heating phases if $\mathcal{C}_{1} \cdot \mathcal{C}_{2}<$ 0 . On the other hand, for $\mathcal{C}_{1} \cdot \mathcal{C}_{2}>0$, one always has $\operatorname{Tr}\left(M_{1} \cdot M_{2}\right)>2$ and there is only a heating phase.

\section{(v) Parabolic-hyperbolic}

Without loss of generality, we consider the case that $H_{1}$ is parabolic, and $H_{2}$ is hyperbolic. Then we have

$$
\begin{aligned}
\operatorname{Tr}\left(M_{1} \cdot M_{2}\right)= & 2 \cosh \left(\frac{\pi \mathcal{C}_{2} T_{2}}{l}\right) \\
& +\frac{2 \mathcal{C}_{1} \cdot \mathcal{C}_{2}}{\mathcal{C}_{2}} \cdot \frac{\pi T_{1}}{l} \cdot \sinh \left(\frac{\pi \mathcal{C}_{2} T_{2}}{l}\right) .
\end{aligned}
$$

Recall that $T_{1}, T_{2}>0$, it is straightforward to check that there are both heating and heating phases if $\mathcal{C}_{1} \cdot \mathcal{C}_{2}<0$. There is only a heating phase if $\mathcal{C}_{1} \cdot \mathcal{C}_{2} \geq 0$.

(vi) Hyperbolic-hyperbolic
If both the driving Hamiltonians are hyperbolic, then we have

$$
\begin{aligned}
\operatorname{Tr}\left(M_{1} \cdot M_{2}\right)= & 2 \cosh \left(\frac{\pi \mathcal{C}_{1} T_{1}}{l}\right) \cdot \cosh \left(\frac{\pi \mathcal{C}_{2} T_{2}}{l}\right) \\
& +\frac{2 \mathcal{C}_{1} \cdot \mathcal{C}_{2}}{\mathcal{C}_{1} \cdot \mathcal{C}_{2}} \cdot \sinh \left(\frac{\pi \mathcal{C}_{1} T_{1}}{l}\right) \cdot \sinh \left(\frac{\pi \mathcal{C}_{2} T_{2}}{l}\right),
\end{aligned}
$$

which may be rewritten as

$$
\begin{aligned}
\operatorname{Tr}\left(M_{1} \cdot M_{2}\right) & =2 \cosh \left(\frac{\pi \mathcal{C}_{1} T_{1}}{l}-\frac{\pi \mathcal{C}_{2} T_{2}}{l}\right) \\
& +2\left(1+\frac{\mathcal{C}_{1} \cdot \mathcal{C}_{2}}{\mathcal{C}_{1} \cdot \mathcal{C}_{2}}\right) \cdot \sinh \left(\frac{\pi \mathcal{C}_{1} T_{1}}{l}\right) \cdot \sinh \left(\frac{\pi \mathcal{C}_{2} T_{2}}{l}\right)
\end{aligned}
$$

For $\frac{\mathcal{C}_{1} \cdot \mathcal{C}_{2}}{\mathcal{C}_{1} \cdot \mathcal{C}_{2}}=-1$, the driven $\mathrm{CFT}$ will stay at the phase transition (or critical phase) along the line

$$
\frac{\mathcal{C}_{1} T_{1}}{l}=\frac{\mathcal{C}_{2} T_{2}}{l}
$$

Away from this critical line, the driven CFT will always be in the heating phase.

For $\frac{\mathcal{C}_{1} \cdot \mathcal{C}_{1}}{\mathcal{C}_{1} \cdot \mathcal{C}_{2}}>-1$, one always has $\operatorname{Tr}\left(M_{1} \cdot M_{2}\right)>2$, and therefore the system is in the heating phase.

The non-heating phase can appear if and only if

$$
\frac{\mathcal{C}_{1} \cdot \mathcal{C}_{1}}{\mathcal{C}_{1} \cdot \mathcal{C}_{2}}<-1
$$

It is interesting to check how the non-heating phases disappear as $\frac{\mathcal{C}_{1} \cdot \mathcal{C}_{1}}{\mathcal{C}_{1} \cdot \mathcal{C}_{2}}$ approaches -1 from the side of $\frac{\mathcal{C}_{1} \cdot \mathcal{C}_{1}}{\mathcal{C}_{1} \cdot \mathcal{C}_{2}}<$ -1 . For convenience, we denote $\frac{\mathcal{C}_{1} \cdot \mathcal{C}_{1}}{\mathcal{C}_{1} \cdot \mathcal{C}_{2}}=-1-\epsilon$, where $\epsilon=0^{+}$. In this limit, Eq. (B9) can be rewritten as

$$
\begin{aligned}
\operatorname{Tr}\left(M_{1} \cdot M_{2}\right) & =2 \cosh \left(\frac{\pi \mathcal{C}_{1} T_{1}}{l}-\frac{\pi \mathcal{C}_{2} T_{2}}{l}\right) \\
& -2 \epsilon \cdot \sinh \left(\frac{\pi \mathcal{C}_{1} T_{1}}{l}\right) \cdot \sinh \left(\frac{\pi \mathcal{C}_{2} T_{2}}{l}\right)
\end{aligned}
$$

One can find that the non-heating phases are composed of three lines connected by a tri-junction (or island), as shown in Fig. 5 for example. This can be understood as follows. First, let us consider the line along that defined in Eq. (B10). By requiring $\operatorname{Tr}\left(M_{1} \cdot M_{2}\right)=0$, one can obtain

$$
\frac{\mathcal{C}_{1} T_{1}}{l}=\frac{\mathcal{C}_{2} T_{2}}{l}=\frac{1}{\pi} \operatorname{arcsinh} \sqrt{\frac{1}{\epsilon}} .
$$

For all $\frac{\mathcal{C}_{1} T_{1}}{l}, \frac{\mathcal{C}_{2} T_{2}}{l}<\frac{1}{\pi} \operatorname{arcsinh} \sqrt{\frac{1}{\epsilon}}$ along the line in Eq. (B10), one has $0<\operatorname{Tr}\left(M_{1} \cdot M_{2}\right)<2$ and therefore the driven CFT is in a non-heating phase. It is noted that as $\epsilon \rightarrow 0^{+}$, Eq. (B13) can be simplified as follows

$$
\frac{\mathcal{C}_{1} T_{1}}{l}=\frac{\mathcal{C}_{1} T_{1}}{l} \simeq \frac{1}{\pi} \log \frac{2}{\epsilon}
$$

The upper boundary of the non-heating phase along the line in (B10) can be obtained by considering $\operatorname{Tr}\left(M_{1}\right.$. $\left.M_{2}\right)=-2$, based on which one can obtain $\frac{\mathcal{C}_{1} T_{1}}{l}=\frac{\mathcal{C}_{2} T_{2}}{l}=$ $\frac{1}{\pi} \operatorname{arcsinh} \sqrt{\frac{1}{\epsilon}}$. 
There are several interesting features: (i) The nonheating phases are composed of three lines connected by an island. (ii) As we approach $\frac{\mathcal{C}_{1}}{\mathcal{C}_{1}} \cdot \frac{\mathcal{C}_{2}}{\mathcal{C}_{2}}=-1$ from the side of $\frac{\mathcal{C}_{1}}{\mathcal{C}_{1}} \cdot \frac{\mathcal{C}_{2}}{\mathcal{C}_{2}}<-1$, the island will move to the infinity.

As a summary of this appendix, for $N=2$ noncommuting driving Hamiltonians, the phase diagram in the parameter space spanned by $T_{1} / l$ and $T_{2} / l$ depends on the Hamiltonian types of both $H_{1}$ and $H_{2}$. If at least one of the two Hamiltonians is elliptic, then there must exist non-heating phases in the phase diagram.

\section{Heating line in the elliptic-elliptic driving}

In this appendix, we show that for two arbitrary noncommuting driving Hamiltonians that are elliptic, there always exist heating lines (lines along which the system is in heating phases) in the phase diagram.

For later use, let us first define the reflection matrix: $M \in \mathrm{SU}(1,1)$ is called reflection if $M^{2}=-\mathbb{I}$ and $\operatorname{Tr}(M)=0^{61}$.

From the definition, a reflection matrix is always elliptic. In addition, it can be proved that the product of two non-commuting reflection matrices is always hyperbolic ${ }^{61}$. In other words, if both $M_{1}$ and $M_{2}$ are reflection matrices, and they do not commute with each other, then we always have $\left|\operatorname{Tr}\left(M_{1} \cdot M_{2}\right)\right|>2$.

Based on the above discussions, we can find that there are always heating phases in the phase diagrams, if both the two non-commuting driving Hamiltonians are of elliptic types. The proof of this claim is as follows:

We consider two non-commuting drivings with $\left(H_{1}, T_{1}\right)$ and $\left(H_{2}, T_{2}\right)$, the corresponding Möbius transformations are expressed in Eq. (2.8). One can find that by choosing

$$
T_{j}=\frac{l}{2 \mathcal{C}_{j}},
$$

where $j=1,2$, one has $\alpha_{j}=-i \frac{\sigma_{j}^{0}}{\mathcal{C}_{j}}, \beta_{j}=-i \frac{\sigma_{j}^{+}+i \sigma_{j}^{-}}{\mathcal{C}_{j}}$, and

$$
\operatorname{Tr}\left(M_{1} \cdot M_{2}\right)=\operatorname{Tr}\left(M_{2} \cdot M_{1}\right)=2 \frac{\mathcal{C}_{1} \cdot \mathcal{C}_{2}}{\mathcal{C}_{1} \mathcal{C}_{2}} .
$$

For non-commuting elliptic Hamiltonians $H_{1}$ and $H_{2}$, one can find that $\left|\operatorname{Tr}\left(M_{1} \cdot M_{2}\right)\right|>2$. This can be understood as follows. One can always find a $\mathrm{SU}(1,1)$ matrix $U$, such that $\operatorname{Tr}\left(U M_{1} U^{-1} \cdot U M_{2} U^{-1}\right)=2 \frac{\mathcal{C}_{1}^{\prime} \cdot \mathcal{C}_{2}^{\prime}}{\mathcal{C}_{1}^{\prime} \mathcal{C}_{2}^{\prime}}$, where the normalized vector $\frac{\mathcal{C}_{1}^{\prime}}{\mathcal{C}_{1}^{\prime}}$ is rotated to $(i, 0,0)$ or $(-i, 0,0)$. Since $M_{2} \neq \pm M_{1}$, we have $\frac{\mathcal{C}_{2}^{\prime}}{\mathcal{C}_{2}}=\left(i \sigma_{2}^{0 \prime}, \sigma_{2}^{+\prime}, \sigma_{2}^{-\prime}\right)$, with $-\left(\sigma_{2}^{0 \prime}\right)^{2}+\left(\sigma_{2}^{+\prime}\right)^{2}+\left(\sigma_{2}^{-\prime}\right)^{2}=-1$. Since at least one of $\sigma_{2}^{+\prime}$ or $\sigma_{2}^{-\prime}$ is nonzero, we always have $\left(\sigma_{2}^{0 \prime}\right)^{2}>1$ (See Fig. 7).

Therefore, $\left|\operatorname{Tr}\left(M_{1} \cdot M_{2}\right)\right|=\left|2 \sigma_{2}^{0 \prime}\right|>2$, or equivalently

$$
\left|\frac{\mathcal{C}_{1} \cdot \mathcal{C}_{2}}{\mathcal{C}_{1} \mathcal{C}_{2}}\right|>1
$$

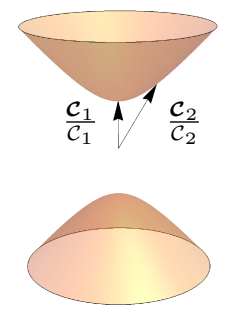

FIG. 7. Two vectors $\frac{\mathcal{C}_{1}}{\mathcal{C}_{1}}$ and $\frac{\mathcal{C}_{2}}{\mathcal{C}_{2}}$ corresponding to two noncommuting reflection matrices $M_{1}$ and $M_{2}$ in (B16).

In short, by choosing two non-commuting Hamtiltonians which are both elliptic, we always have a heating phase at the point $\left(T_{1}, T_{2}\right)=\left(\frac{l}{2 \mathcal{C}_{1}}, \frac{l}{2 \mathcal{C}_{2}}\right)$.

Now we will show that there always exits a 'heating line' in the phase diagram if both driving Hamiltonians are elliptic.

Now we only focus on a 'unit cell' with $0<T_{j} \leq l / \mathcal{C}_{j}$ $(j=1,2)$ in the phase diagram. The locations of heating line depends on the sign of $\mathcal{C}_{1} \cdot \mathcal{C}_{2}$ as follows:

1. If $\mathcal{C}_{1} \cdot \mathcal{C}_{2}<0$, the heating line is determined by

$$
\frac{T_{1}}{l_{1, \mathrm{eff}}}+\frac{T_{2}}{l_{2, \mathrm{eff}}}=1, \quad 0<T_{1}<l_{1, \mathrm{eff}},
$$

where we have defined $l_{i \text {,eff }}=l / \mathcal{C}_{i}$ in the elliptic case.

2. If $\mathcal{C}_{1} \cdot \mathcal{C}_{2}>0$, the heating line is determined by

$$
\frac{T_{1}}{l_{1, \mathrm{eff}}}-\frac{T_{2}}{l_{2, \mathrm{eff}}}=0, \quad 0<T_{1}<l_{1, \mathrm{eff}} .
$$

Examples corresponding to these two cases can be found in Fig. 8. Now we give the proofs of these two claims as follows.

Let us consider $\mathcal{C}_{1} \cdot \mathcal{C}_{2}<0$ first. Based on Eqs. (B18) and (B1) one can find that

$$
\operatorname{Tr}\left(M_{1} \cdot M_{2}\right)=-2 \cos ^{2}\left(\frac{\pi T_{1}}{l_{1, \mathrm{eff}}}\right)+2 \frac{\mathcal{C}_{1} \cdot \mathcal{C}_{2}}{\mathcal{C}_{1} \mathcal{C}_{2}} \cdot \sin ^{2}\left(\frac{\pi T_{1}}{l_{1, \mathrm{eff}}}\right)
$$

Since $\mathcal{C}_{1} \cdot \mathcal{C}_{2}<0$, we have $\frac{\mathcal{C}_{1} \cdot \mathcal{C}_{2}}{\mathcal{C}_{1} \mathcal{C}_{2}}<-1$ based on Eq. (B17). Then one can find

$$
\operatorname{Tr}\left(M_{1} \cdot M_{2}\right)=-2+2\left(\frac{\mathcal{C}_{1} \cdot \mathcal{C}_{2}}{\mathcal{C}_{1} \mathcal{C}_{2}}+1\right) \cdot \sin ^{2}\left(\frac{\pi T_{1}}{l_{1, \text { eff }}}\right)<-2 .
$$

Therefore, we always have a heating phase along the line defined in Eq. (B18).

Second, let us consider $\mathcal{C}_{1} \cdot \mathcal{C}_{2}>0$. Based on Eqs. (B19) and (B1) one can find that

$$
\operatorname{Tr}\left(M_{1} \cdot M_{2}\right)=2+2\left(\frac{\mathcal{C}_{1} \cdot \mathcal{C}_{2}}{\mathcal{C}_{1} \mathcal{C}_{2}}-1\right) \cdot \sin ^{2}\left(\frac{\pi T_{1}}{l_{1, \text { eff }}}\right)>2,
$$

where we have considered $\mathcal{C}_{1} \cdot \mathcal{C}_{2}>0$ and therefore $\frac{\mathcal{C}_{1} \cdot \mathcal{C}_{2}}{\mathcal{C}_{1} \mathcal{C}_{2}}>1$ based on Eq. (B17).

Till now, we have proved that the lines in Eq. (B18) for $\mathcal{C}_{1} \cdot \mathcal{C}_{2}<0$ and those in Eq. (B19) for $\mathcal{C}_{1} \cdot \mathcal{C}_{2}>0$ are always in the heating phase. 

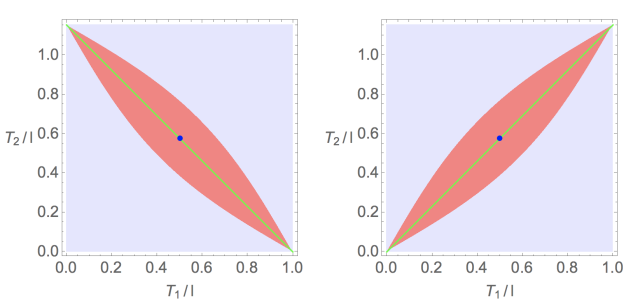

FIG. 8. Heating line (in green) determined by Eqs.(B18) and (B19) in the unit cell with $0<T_{j} \leq l / \mathcal{C}_{j}(j=1,2)$ in the cases of $\mathcal{C}_{1}=(1,0,0)$ and $\mathcal{C}_{2}=(1,0.5,0)$ (left) and $\mathcal{C}_{1}=(1,0,0)$ and $\mathcal{C}_{2}=(-1,0.5,0)$ (right). Here we choose $l=1$. The blue dots represent the heating point as defined in Eq.(B15). The regions in red (blue) corresponds to a heating (non-heating) phase.

\section{Appendix C: $N=3$}

As discussed in Sec. III C, when all the three driving Hamiltonians are non-elliptic, the intrinsic- $N(N=3)$ condition is $\eta_{N}<0$ in (3.14). In the main text, we consider the cases with (i) three parabolic Hamiltonians and (ii) three hyperbolic Hamiltonians. In this appendix, we consider the other two cases, i.e., (iii) one parabolic and two hyperbolic Hamiltonians and (iv) two parabolic and one hyperbolic Hamiltonians.

- 1 parabolic and 2 hyperbolic Hamiltonians

Now let us consider the case there are one parabolic and two hyperbolic driving Hamiltonians. Without loss of generality, let us choose $H_{1}$ is parabolic, and $H_{2}, H_{3}$ are hyperbolic. Based on Eqs. (2.9) and (2.10), one has

$$
\begin{aligned}
& \operatorname{Tr}\left(M_{1} \cdot M_{2} \cdot M_{3}\right)=2 \cosh \left(\frac{\pi \mathcal{C}_{2} T_{2}}{l}\right) \cosh \left(\frac{\pi \mathcal{C}_{3} T_{3}}{l}\right) \\
& +2 \frac{\mathcal{C}_{1} \cdot \mathcal{C}_{3}}{\mathcal{C}_{3}} \cdot \frac{\pi T_{1}}{l} \cdot \cosh \left(\frac{\pi \mathcal{C}_{2} T_{2}}{l}\right) \sinh \left(\frac{\pi \mathcal{C}_{3} T_{3}}{l}\right) \\
& +2 \frac{\mathcal{C}_{1} \cdot \mathcal{C}_{2}}{\mathcal{C}_{2}} \cdot \frac{\pi T_{1}}{l} \cdot \cosh \left(\frac{\pi \mathcal{C}_{3} T_{3}}{l}\right) \sinh \left(\frac{\pi \mathcal{C}_{2} T_{2}}{l}\right) \\
& +2 \frac{\mathcal{C}_{2} \cdot \mathcal{C}_{3}}{\mathcal{C}_{2} \mathcal{C}_{3}} \cdot \sinh \left(\frac{\pi \mathcal{C}_{2} T_{2}}{l}\right) \sinh \left(\frac{\pi \mathcal{C}_{3} T_{3}}{l}\right) \\
& +2 \frac{\mathcal{C}_{1} * \mathcal{C}_{2} * \mathcal{C}_{3}}{\mathcal{C}_{2} \mathcal{C}_{3}} \cdot \frac{\pi T_{1}}{l} \cdot \sinh \left(\frac{\pi \mathcal{C}_{2} T_{2}}{l}\right) \sinh \left(\frac{\pi \mathcal{C}_{3} T_{3}}{l}\right) .
\end{aligned}
$$

The intrinsic- $N$ condition in (3.14) can be understood as follows. For $\frac{T_{i}}{l} \rightarrow 0$, one has $\operatorname{Tr}\left(M_{1} \cdot M_{2} \cdot M_{3}\right) \simeq 2$. On the other hand, by taking the limit $\frac{T_{i}}{l} \rightarrow \infty$, one has $\operatorname{Tr}\left(M_{1}\right.$. $\left.M_{2} \cdot M_{3}\right) \simeq \eta_{3} \cdot \frac{\pi T_{1}}{l} \cosh \left(\frac{\pi \mathcal{C}_{2} T_{2}}{l}\right) \cosh \left(\frac{\pi \mathcal{C}_{3} T_{3}}{l}\right) \rightarrow-\infty$ for $\eta_{3}<0$, where we have considered $\cosh \frac{\pi \mathcal{C}_{i} T_{i}}{l} \simeq \sinh \frac{\pi \mathcal{C}_{i} T_{i}}{l}$ and $\eta_{3}=2 \frac{\mathcal{C}_{1} \cdot \mathcal{C}_{3}}{\mathcal{C}_{3}}+2 \frac{\mathcal{C}_{1} \cdot \mathcal{C}_{2}}{\mathcal{C}_{2}}+2 \frac{\mathcal{C}_{1} * \mathcal{C}_{2} * \mathcal{C}_{3}}{\mathcal{C}_{2} \mathcal{C}_{3}}$. Here one can check explicitly that $\eta_{3}=\operatorname{Tr}\left(P_{1} \cdot P_{2} \cdot P_{3}\right)$, with $P_{i}$ expressed in (3.5) and (3.6). Then as we tune the parameters $\frac{T_{i}}{l}$ continuously, there must exist non-heating phases with $\left|\operatorname{Tr}\left(M_{1} \cdot M_{2} \cdot M_{3}\right)\right|<2$.

\section{- 2 parabolic and 1 hyperbolic Hamiltonians}

Now let us consider the case with two parabolic and one hyperbolic driving Hamiltonians. Without loss of generality, let us choose $H_{1}$ and $H_{2}$ to be parabolic, and $H_{3}$ to be hyperbolic. Based on Eqs. (2.9) and (2.10), one has

$$
\begin{aligned}
& \operatorname{Tr}\left(M_{1} \cdot M_{2} \cdot M_{3}\right)=2 \cosh \left(\frac{\pi \mathcal{C}_{3} T_{3}}{l}\right) \\
& +2 \frac{\mathcal{C}_{1} \cdot \mathcal{C}_{3}}{\mathcal{C}_{3}} \cdot \frac{\pi T_{1}}{l} \cdot \sinh \left(\frac{\pi \mathcal{C}_{3} T_{3}}{l}\right) \\
& +2 \frac{\mathcal{C}_{2} \cdot \mathcal{C}_{3}}{\mathcal{C}_{3}} \cdot \frac{\pi T_{2}}{l} \cdot \sinh \left(\frac{\pi \mathcal{C}_{3} T_{3}}{l}\right) \\
& +2 \mathcal{C}_{1} \cdot \mathcal{C}_{2} \cdot \frac{\pi T_{1}}{l} \cdot \frac{\pi T_{2}}{l} \cdot \cosh \left(\frac{\pi \mathcal{C}_{3} T_{3}}{l}\right) \\
& +2 \frac{\mathcal{C}_{1} * \mathcal{C}_{2} * \mathcal{C}_{3}}{\mathcal{C}_{3}} \cdot \frac{\pi T_{1}}{l} \cdot \frac{\pi T_{2}}{l} \cdot \sinh \left(\frac{\pi \mathcal{C}_{3} T_{3}}{l}\right)
\end{aligned}
$$

As before, in the limit $\frac{T_{i}}{l} \rightarrow 0$, one has $\operatorname{Tr}\left(M_{1} \cdot M_{2} \cdot M_{3}\right) \simeq$ 2. In the other limit $\frac{T_{i}}{l} \rightarrow \infty$, one has $\operatorname{Tr}\left(M_{1} \cdot M_{2}\right.$. $\left.M_{3}\right) \simeq \eta_{3} \cdot \frac{\pi T_{1}}{l} \cdot \frac{\pi T_{2}}{l} \cosh \left(\frac{\pi \mathcal{C}_{3} T_{3}}{l}\right) \rightarrow-\infty$ for $\eta_{3}<0$, where we have considered $\cosh \frac{\pi \mathcal{C}_{i} T_{i}}{l} \simeq \sinh \frac{\pi \mathcal{C}_{i} T_{i}}{l}$ and $\eta_{3}=2 \mathcal{C}_{1} \cdot \mathcal{C}_{2}+2 \frac{\mathcal{C}_{1} * \mathcal{C}_{2} * \mathcal{C}_{3}}{\mathcal{C}_{2} \mathcal{C}_{3}}$. Here one can check explicitly that $\eta_{3}=\operatorname{Tr}\left(P_{1} \cdot P_{2} \cdot P_{3}\right)$, with $P_{i}$ expressed in (3.5) and (3.6). Apparently, as we tune the parameters $\frac{T_{i}}{l}$ from 0 to $\infty$, there exist non-heating phases with $\mid \operatorname{Tr}\left(M_{1} \cdot M_{2}\right.$. $\left.M_{3}\right) \mid<2$. 
1 Liang Jiang, Takuya Kitagawa, Jason Alicea, A. R. Akhmerov, David Pekker, Gil Refael, J. Ignacio Cirac, Eugene Demler, Mikhail D. Lukin, and Peter Zoller, "Majorana Fermions in Equilibrium and Driven Cold Atom Quantum Wires," Phys. Rev. Lett. 106, 220402 (2011), arXiv:1102.5367 [cond-mat.quant-gas].

2 Takuya Kitagawa, Erez Berg, Mark Rudner, and Eugene Demler, "Topological characterization of periodically driven quantum systems," Phys. Rev. B 82, 235114 (2010).

3 Mark S. Rudner, Netanel H. Lindner, Erez Berg, and Michael Levin, "Anomalous edge states and the bulk-edge correspondence for periodically driven two-dimensional systems," Phys. Rev. X 3, 031005 (2013).

${ }^{4}$ Dominic V. Else and Chetan Nayak, "Classification of topological phases in periodically driven interacting systems," Phys. Rev. B 93, 201103 (2016).

5 Andrew C. Potter, Takahiro Morimoto, and Ashvin Vishwanath, "Classification of interacting topological floquet phases in one dimension," Phys. Rev. X 6, 041001 (2016).

6 Rahul Roy and Fenner Harper, "Abelian floquet symmetry-protected topological phases in one dimension," Phys. Rev. B 94, 125105 (2016).

7 Hoi Chun Po, Lukasz Fidkowski, Takahiro Morimoto, Andrew C. Potter, and Ashvin Vishwanath, "Chiral Floquet Phases of Many-Body Localized Bosons," Phys. Rev. X6, 041070 (2016), arXiv:1609.00006 [cond-mat.dis-nn].

8 Rahul Roy and Fenner Harper, "Periodic table for floquet topological insulators," Phys. Rev. B 96, 155118 (2017).

9 Fenner Harper and Rahul Roy, "Floquet topological order in interacting systems of bosons and fermions," Phys. Rev. Lett. 118, 115301 (2017).

10 Hoi Chun Po, Lukasz Fidkowski, Ashvin Vishwanath, and Andrew C. Potter, "Radical chiral floquet phases in a periodically driven kitaev model and beyond," Phys. Rev. B 96, 245116 (2017).

11 I.-D. Potirniche, A. C. Potter, M. Schleier-Smith, A. Vishwanath, and N. Y. Yao, "Floquet symmetry-protected topological phases in cold-atom systems," Phys. Rev. Lett. 119, 123601 (2017).

12 Takahiro Morimoto, Hoi Chun Po, and Ashvin Vishwanath, "Floquet topological phases protected by time glide symmetry," Phys. Rev. B 95, 195155 (2017).

13 Lukasz Fidkowski, Hoi Chun Po, Andrew C. Potter, and Ashvin Vishwanath, "Interacting invariants for floquet phases of fermions in two dimensions," Phys. Rev. B 99, 085115 (2019).

14 Paolo Glorioso, Andrey Gromov, and Shinsei Ryu, "Effective response theory for floquet topological systems," (2019), arXiv:1908.03217 [cond-mat.str-el].

15 Vedika Khemani, Achilleas Lazarides, Roderich Moessner, and S. L. Sondhi, "Phase structure of driven quantum systems," Phys. Rev. Lett. 116, 250401 (2016).

16 Dominic V. Else, Bela Bauer, and Chetan Nayak, "Floquet time crystals," Phys. Rev. Lett. 117, 090402 (2016).

17 C. W. von Keyserlingk and S. L. Sondhi, "Phase structure of one-dimensional interacting floquet systems. i. abelian symmetry-protected topological phases," Phys. Rev. B 93, 245145 (2016).

18 C. W. von Keyserlingk and S. L. Sondhi, "Phase structure of one-dimensional interacting floquet systems. ii. symmetry-broken phases," Phys. Rev. B 93, 245146
(2016).

19 Dominic V. Else, Bela Bauer, and Chetan Nayak, "Prethermal Phases of Matter Protected by TimeTranslation Symmetry," Phys. Rev. X7, 011026 (2017), arXiv:1607.05277 [cond-mat.stat-mech].

20 N. Y. Yao, A. C. Potter, I.-D. Potirniche, and A. Vishwanath, "Discrete time crystals: Rigidity, criticality, and realizations," Phys. Rev. Lett. 118, 030401 (2017).

21 Soonwon Choi, Joonhee Choi, Renate Landig, Georg Kucsko, Hengyun Zhou, Junichi Isoya, Fedor Jelezko, Shinobu Onoda, Hitoshi Sumiya, Vedika Khemani, et al., "Observation of discrete time-crystalline order in a disordered dipolar many-body system," Nature 543, 221 (2017), 1610.08057.

22 J. Zhang, P. W. Hess, A. Kyprianidis, P. Becker, A. Lee, J. Smith, G. Pagano, I. D. Potirniche, A. C. Potter, A. Vishwanath, N. Y. Yao, and C. Monroe, "Observation of a discrete time crystal," Nature 543, 217-220 (2017), arXiv:1609.08684 [quant-ph].

23 Norman Y. Yao, Chetan Nayak, Leon Balents, and Michael P. Zaletel, "Classical Discrete Time Crystals," arXiv e-prints , arXiv:1801.02628 (2018), arXiv:1801.02628 [cond-mat.stat-mech].

24 Luca D'Alessio and Marcos Rigol, "Long-time Behavior of Isolated Periodically Driven Interacting Lattice Systems," Physical Review X 4, 041048 (2014), arXiv:1402.5141 [cond-mat.stat-mech].

25 Pedro Ponte, Z. Papic, Francois Huveneers, and Dmitry A. Abanin, "Many-Body Localization in Periodically Driven Systems," PRL 114, 140401 (2015), arXiv:1410.8518 [cond-mat.dis-nn].

26 Dmitry A. Abanin, Wojciech De Roeck, and Francois Huveneers, "Theory of many-body localization in periodically driven systems," Annals of Physics 372, 1-11 (2016), arXiv:1412.4752 [cond-mat.dis-nn].

27 Dmitry A. Abanin, Wojciech De Roeck, and Francois Huveneers, "Exponentially Slow Heating in Periodically Driven Many-Body Systems," PRL 115, 256803 (2015), arXiv:1507.01474 [cond-mat.stat-mech].

28 Dmitry Abanin, Wojciech De Roeck, Wen Wei Ho, and Francois Huveneers, "A Rigorous Theory of Many-Body Prethermalization for Periodically Driven and Closed Quantum Systems," Communications in Mathematical Physics 354, 809-827 (2017), arXiv:1509.05386 [math-ph].

29 Dmitry A. Abanin, Wojciech De Roeck, Wen Wei Ho, and Francois Huveneers, "Effective Hamiltonians, prethermalization, and slow energy absorption in periodically driven many-body systems," PRB 95, 014112 (2017), arXiv:1510.03405 [cond-mat.stat-mech].

30 C. K. Law, "Resonance response of the quantum vacuum to an oscillating boundary," Phys. Rev. Lett. 73, 1931-1934 (1994)

31 V. V. Dodonov and A. B. Klimov, "Generation and detection of photons in a cavity with a resonantly oscillating boundary," Phys. Rev. A 53, 2664-2682 (1996).

32 Ivar Martin, "Floquet dynamics of classical and quantum cavity fields," Annals of Physics 405, 101 - 129 (2019).

33 Alexander A Belavin, Alexander M Polyakov, and Alexander B Zamolodchikov, "Infinite conformal symmetry in two-dimensional quantum field theory," Nuclear Physics B 241, 333-380 (1984). 
34 Daniel Friedan, Zongan Qiu, and Stephen Shenker, "Conformal invariance, unitarity, and critical exponents in two dimensions," Phys. Rev. Lett. 52, 1575-1578 (1984).

35 Philippe Francesco, Pierre Mathieu, and David Sénéchal, Conformal field theory (Springer Science \& Business Media, 2012).

36 Pasquale Calabrese and John Cardy, "Evolution of entanglement entropy in one-dimensional systems," Journal of Statistical Mechanics: Theory and Experiment 2005, P04010 (2005).

37 Pasquale Calabrese and John Cardy, "Time dependence of correlation functions following a quantum quench," Phys. Rev. Lett. 96, 136801 (2006).

38 Xueda Wen and Jie-Qiang Wu, "Quantum dynamics in sine-square deformed conformal field theory: Quench from uniform to nonuniform conformal field theory," Phys. Rev. B97, 184309 (2018), arXiv:1802.07765 [cond-mat.str-el].

39 Xueda Wen and Jie-Qiang Wu, "Floquet conformal field theory," (2018), arXiv:1805.00031 [cond-mat.str-el].

40 Toshiya Hikihara and Tomotoshi Nishino, "Connecting distant ends of one-dimensional critical systems by a sinesquare deformation," Phys. Rev. B 83, 060414 (2011).

41 Isao Maruyama, Hosho Katsura, and Toshiya Hikihara, "Sine-square deformation of free fermion systems in one and higher dimensions," PRB 84, 165132 (2011), arXiv:1108.2973 [cond-mat.stat-mech].

42 Hosho Katsura, "Sine-square deformation of solvable spin chains and conformal field theories," Journal of Physics A: Mathematical and Theoretical 45, 115003 (2012).

43 Nobuyuki Ishibashi and Tsukasa Tada, "Infinite circumference limit of conformal field theory," Journal of Physics A: Mathematical and Theoretical 48, 315402 (2015).

44 Nobuyuki Ishibashi and Tsukasa Tada, "Dipolar quantization and the infinite circumference limit of two-dimensional conformal field theories," International Journal of Modern Physics A 31, 1650170 (2016).

45 Kouichi Okunishi, "Sine-square deformation and MÃ $q$ bius quantization of 2D conformal field theory," PTEP 2016, 063A02 (2016), arXiv:1603.09543 [hep-th].

46 Xueda Wen, Shinsei Ryu, and Andreas W. W. Ludwig, "Evolution operators in conformal field theories and conformal mappings: Entanglement Hamiltonian, the sinesquare deformation, and others," Phys. Rev. B93, 235119 (2016), arXiv:1604.01085 [cond-mat.str-el].

47 Shota Tamura and Hosho Katsura, "Zero-energy states in conformal field theory with sine-square deformation," PTEP 2017, 113A01 (2017), arXiv:1709.06238 [condmat.stat-mech].

48 Tsukasa Tada, "Conformal Quantum Mechanics and Sine-Square Deformation," PTEP 2018, 061B01 (2018), arXiv:1712.09823 [hep-th].

49 Pawel Caputa and Ian MacCormack, "Geometry and complexity of path integrals in inhomogeneous cfts," (2020), arXiv:2004.04698 [hep-th].

${ }^{50}$ Xun Liu and Tsukasa Tada, "Analysis for lorentzian conformal field theories through sine-square deformation," (2020), arXiv:2004.01930 [hep-th].

51 Tsukasa Tada, "Time development of conformal field theories associated with $l_{1}$ and $l_{-1}$ operators," (2019), arXiv:1904.12414 [hep-th].

${ }^{52}$ Ian MacCormack, Aike Liu, Masahiro Nozaki, and Shinsei Ryu, "Holographic duals of inhomogeneous systems: The rainbow chain and the sine-square deformation model," Journal of Physics A: Mathematical and Theoret- ical (2019).

53 Ruihua Fan, Yingfei Gu, Ashvin Vishwanath, and Xueda Wen, "Emergent spatial structure and entanglement localization in floquet conformal field theory," (2019), arXiv:1908.05289 [cond-mat.str-el].

54 Bastien Lapierre, Kenny Choo, Clément Tauber, Apoorv Tiwari, Titus Neupert, and Ramasubramanian Chitra, "Emergent black hole dynamics in critical floquet systems," Phys. Rev. Research 2, 023085 (2020).

55 Xueda Wen, Ruihua Fan, Ashvin Vishwanath, and Yingfei $\mathrm{Gu}$, "Periodically, quasi-periodically, and randomly driven conformal field theories: Part i," (2020), arXiv:2006.10072 [cond-mat.stat-mech].

56 Bastien Lapierre, Kenny Choo, Apoorv Tiwari, Clement Tauber, Titus Neupert, and Ramasubramanian Chitra, "The fine structure of heating in a quasiperiodically driven critical quantum system," (2020), arXiv:2006.10054 [condmat.str-el].

57 Xueda Wen, Ruihua Fan, Yingfei Gu, and Ashvin Vishwanath, "Periodically, quasi-periodically, and randomly driven conformal field theories: Part ii," To appear.

58 Edward Witten, "Coadjoint orbits of the virasoro group," Comm. Math. Phys. 114, 1-53 (1988).

59 Ruihua Fan, Xueda Wen, Yingfei Gu, and Ashvin Vishwanath, To appear.

60 Bo Han, "More on the generally sl(2) deformed floquet conformal field theories," To appear.

61 Barry Simon, Orthogonal polynomials on the unit circle (American Mathematical Soc., 2005).

62 Pasquale Calabrese and John L. Cardy, "Entanglement entropy and quantum field theory," J. Stat. Mech. 0406, P06002 (2004), arXiv:hep-th/0405152 [hep-th].

63 Pasquale Calabrese and John Cardy, "Entanglement entropy and conformal field theory," Journal of Physics A: Mathematical and Theoretical 42, 504005 (2009).

64 Dmitry S. Ageev, Andrey A. Bagrov, and Askar A. Iliasov, "Deterministic chaos and fractal entropy scaling in floquet cft," (2020), arXiv:2006.11198 [cond-mat.stat-mech].

65 John Cardy and Erik Tonni, "Entanglement hamiltonians in two-dimensional conformal field theory," Journal of Statistical Mechanics: Theory and Experiment 2016, 123103 (2016).

66 Xueda Wen, Shinsei Ryu, and Andreas W W Ludwig, "Entanglement hamiltonian evolution during thermalization in conformal field theory," Journal of Statistical Mechanics: Theory and Experiment 2018, 113103 (2018).

67 Giuseppe Di Giulio, Raúl Arias, and Erik Tonni, "Entanglement hamiltonians in 1d free lattice models after a global quantum quench," Journal of Statistical Mechanics: Theory and Experiment 2019, 123103 (2019).

68 W. Zhu, Zhoushen Huang, Yin-Chen He, and Xueda Wen, "Entanglement hamiltonian of many-body dynamics in strongly correlated systems," Phys. Rev. Lett. 124, 100605 (2020). 